\title{
Determination of Fluorescence Polarization and Absorption Anisotropy in Molecular Complexes Having Threefold Rotational Symmetry
}

\author{
Andrey A. Demidov and David L. Andrews* \\ University of East Anglia, School of Chemical Sciences, Norwich, UK
}

Received 9 March 1995; accepted 15 September 1995

\section{ABSTRACT}

The current work concerns investigation of the polarization properties of complex molecular ensembles exhibiting threefold $\left(\mathrm{C}_{3}\right)$ rotational symmetry, particularly with regard to the interplay between their structure and dynamics of internal energy transfer. We assume that the molecules or chromophores in such complexes possess strongly overlapped spectra both for absorption and fluorescence. Such trimeric structures are widely found in biological preparations, as for example the trimer of $\mathrm{C}$ phycocyanin (C-PC). Higher order aggregates, e.g. hexamers and three-hexamer rods, are also investigated and compared with the trimer case. The theory addresses both steady-state and $\delta$-pulse excitation and establishes some links between them. Monochromophoric, bichromophoric and trichromophoric molecular complexes are individually examined.

For steady-state excitation, analytical formulas are reported for the degree of fluorescence polarization and absorption anisotropy. It is shown that the polarization is dependent on the chromophore inclination relative to the symmetry axis, the relative efficiencies of absorption and fluorescence by chromophores of different spectral types, and the rates of energy equilibration. To assess the validity of the theory, it has been applied to $\mathrm{C}-\mathrm{PC}$ aggregates. Here it was found that different $\mathrm{C}$-PC aggregates provide practically identical polarization response. For $\boldsymbol{\delta}$-pulse excitation we give analytical formulas for determination of the fluorescence depolarization, and also the depolarization associated with absorption recovery, both for a monochromophoric trimer and some particular cases of bichromophoric trimer. More complicated systems are analyzed by computer modeling. Thus it transpires that the initial polarization anisotropy $r(t=0)$ takes the value 0.4 for all considered aggregates; the long-time limit $\mathbf{r}(\mathbf{t} \rightarrow \infty)$ has about the same value as is associated with steady-state excitation. We also show that with steady-state excitation the degree of fluorescence polarization is practically equal for various $C_{3}$ aggregates of $\mathrm{C}-\mathrm{PC}$, and that the major factor determining the polarization is the chromophore orientation relative to the symmetry axis.

C) 1996 American Society for Photobiology 0031-8655/96 \$5.00+0.00

\section{INTRODUCTION}

The impressive efforts of nature in creating photosynthetic complexes with the extraordinarily high efficiency of solar energy harvesting continuously challenges scientists with the problem of how the molecular structure was created and how it works. Polarization spectroscopy provides one of the most promising routes for this quest because fluorescence polarization and absorption anisotropy are sensitive to spectral features and the mutual orientation of molecules within such complexes. This type of technique thus makes it possible both to analyze the structure of salient molecular complexes and to conduct a quantitative investigation of their internal energy transfer processes (1-5).

The classical basis of polarization spectroscopy was developed in the 1920 s by Levshin $(6)$ and Perrin $(7,8)$. The availability of picosecond and femtosecond lasers has now opened up new opportunities for the investigation of energy migration in molecular complexes, and it imparts a new interest to polarization spectroscopy both in experiment (912 ) and theory $(13-17)$. In this connection a general theory of coherent energy transfer has been developed in works by Rahman et al. (13), Wynne and Hochstrasser (14), Knox and Güllen (15) and van Amerongen and Struve $(16,17)$. These studies have led to the result that at time $t=0$ the polarization anisotropy $r(0)$ may be as large as 0.7 when randomly oriented molecular complexes are excited by $\delta$-pulse laser light, and this anisotropy has a decay time typically measured in hundreds of femtoseconds. The theory of incoherent energy transfer $(18-22)$ yields the value $\mathbf{r}(0)=0.4$. Recent experimental results have determined $\mathrm{r}(0)=0.4$ for C-phycocyanin $(C-P C)^{+}$trimers $(10,12)$, allophycocyanin (APC) trimers $(12,23)$, photosystem I (PSI) particles $(11)$ and $r(0)$ $=0.7$ for the magnesium derivatives of tetraphenylporphyrin (9).

The case of steady-state excitation is less attractive to theorists, whereas it is more widely used in practical applications of polarization spectroscopy because of its simpler experimental realization. Thus, there is a lack of appropriate theory to describe polarization phenomena in molecular complexes under steady-state excitation. However such

\footnotetext{
*To whom correspondence should be addressed at: University of

East Anglia, School of Chemical Sciences, Norwich NR4 7TJ,

U.K. Fax: 44-(1)603-259855, e-mail: D.L.Andrews@uea.ac.uk. $\dagger$ Abbreviations: APC, allophycocyanin; PC, phycocyanin.
} 


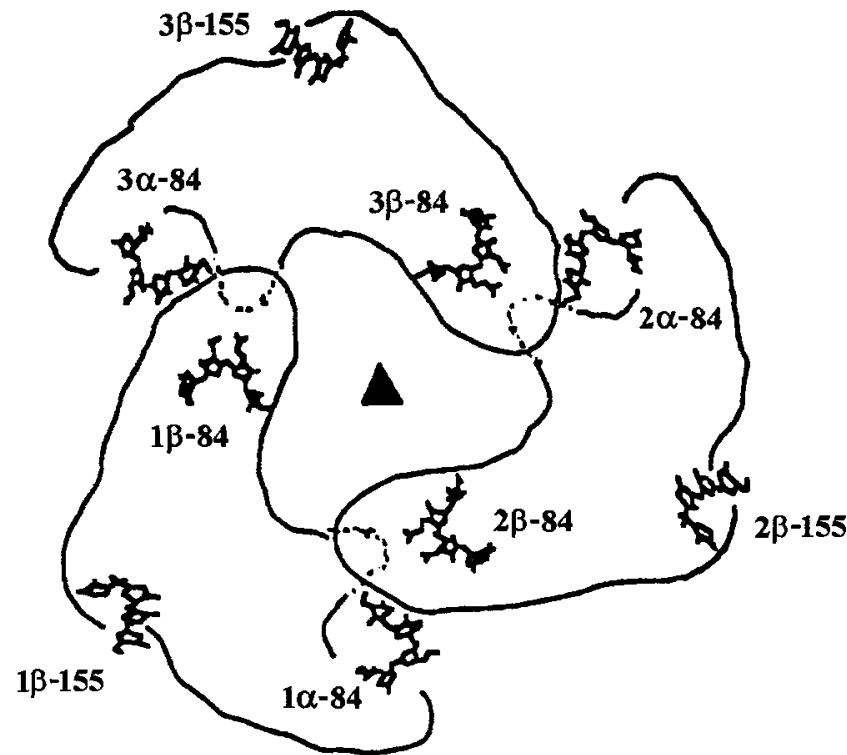

Figure 1. Scheme of the C-PC trimer (redrawn from Xie et al. (12)). This trimer consists of three monomers each having three types of chromophore: $\alpha-84, \beta-84$ and $\beta-155$. The estimated distance (37) between the $\alpha-84$ and $\beta-84$ chromophores of the neighboring monomers is about $20 \AA$.

problems have recently begun to attract new attention $(16,19-21,24)$.

Our current work is devoted to determination of the degree of fluorescence polarization and absorption anisotropy in molecular complexes having threefold $\left(\mathrm{C}_{3}\right)$ rotational symmetry, in the cases of steady-state and $\delta$-pulse excitation. We consider the particular case of complexes containing chromophores of three distinct spectral types, for example C-PC aggregates $(25,26)$, which additionally have an overall $\mathrm{C}_{3}$ symmetry. A functional unit of $\mathrm{C}-\mathrm{PC}$ is a trimer consisting of three identical monomers for which, as shown in Fig. 1 , the threefold symmetry means invariance under rotation of the trimer by $120^{\circ}$. Each monomer consists of $\alpha$ and $\beta$ subunits, which contain the chromophore phycocyanobilin, the open tetrapyrrole ring, bound to the 84th cysteine residue of the $\alpha$-subunit (hereafter called $\alpha-84$ ), and the 84 th and 155 th cysteine residues of the $\beta$-subunit ( $\beta-84$ and $\beta-155)$.

The spatial symmetry of such molecular complexes creates a basis for significant simplification of the theoretical calculations. In particular, it leads to certain results expressed mainly in terms of the chromophore orientation relative to the symmetry axis (see the simplified case presented in Fig. 2) and the orientation of this axis relative to the polarization of incident light.

\section{METHODS}

Let us consider an individual molecular complex with threefold symmetry, like the C-PC trimer, and within it some particular chromophore i. Such a complex is illuminated by polarized light. Figure 3 shows the relative orientations of the unit polarization vector $\mathbf{e}$ of the incident light and the unit vector $\mathbf{i}$ of the $\mathrm{i}$-chromophore transition dipole moment. In the axis frame, fixed relative to the molecular complex, the Cartesian components of the two vectors are $\mathbf{i}=\left\{\sin \theta_{\mathrm{i}} \cos \phi_{\mathrm{i}}, \sin \theta_{\mathrm{i}} \sin \phi_{\mathrm{i}}, \cos \theta_{\mathrm{i}}\right\}$ and $\mathbf{e}=\left\{\sin \theta_{\mathrm{e}} \cos \phi_{\mathrm{e}}\right.$, $\left.\sin \theta_{\mathrm{e}} \sin \phi_{\mathrm{e}}, \cos \theta_{\mathrm{e}}\right\}$.

The efficiency of energy absorption by chromophore $i$ is express-
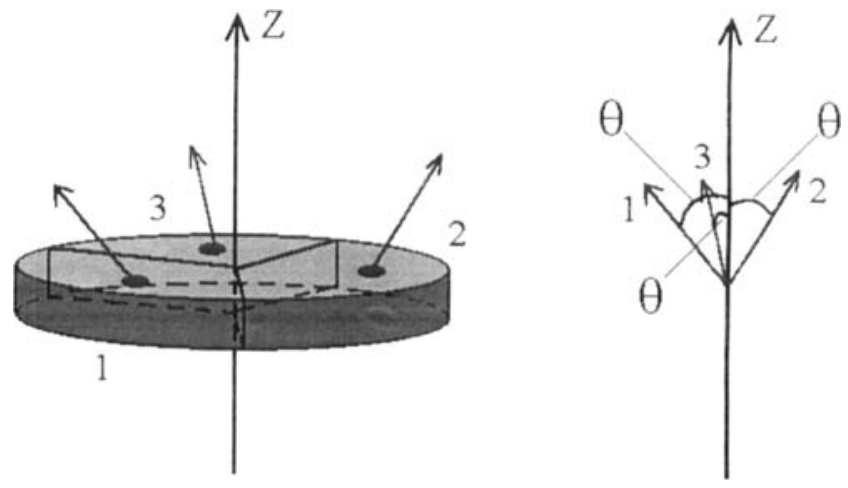

Figure 2. Schematic structure of the monochromophoric trimer. Each monomer contains one chromophore, the indexes 1, 2, 3 denote both the monomer number and the affiliation of the corresponding chromophore. All chromophores have transition dipole moments with equal inclination (angle $\theta$ ) to the axis of symmetry $Z$.

ible as $A^{i}=C \sigma_{a}\left(\lambda_{a}\right)(i, e)^{2}=C \sigma_{i}\left(\lambda_{a}\right)\left\{\sin \theta_{i} \sin \theta_{e} \cos \left(\phi_{i}-\phi_{e}\right)+\cos \right.$ $\left.\theta_{\mathrm{i}} \cos \theta_{\mathrm{e}}\right\}^{2}$, where $(\mathbf{i}, \mathbf{e})$ means the scalar product of the $\mathbf{i}$ and e vectors; $\sigma_{i}\left(\lambda_{a}\right)$ is the absorption cross section of chromophore $i$ at wavelength $\lambda_{\mathrm{a}} ; \mathrm{C}$ is a constant that will disappear in the final expressions. Let us suppose that chromophore $i$ is of the first spectroscopic type. In the trimer we can count another two chromophores of the same type, positioned in the adjoining monomers. The orientation angles of these chromophores are equal in $\theta_{i}$ and differ by $\pm 120^{\circ}$ in the $\phi_{i}$. This yields the following simple expression for the efficiency of energy absorption by all three chromophores of the first spectroscopic type:

$$
\begin{aligned}
A_{1} & =\operatorname{Co}\left(\lambda_{a}\right) \sum_{i=1}^{3} A^{i} \\
& =3 \operatorname{Co} \sigma_{1}\left(\lambda_{a}\right)\left(\cos ^{2} \theta_{1} \cos ^{2} \theta_{e}+0.5 \sin ^{2} \theta_{1} \sin ^{2} \theta_{e}\right)
\end{aligned}
$$

where the subscript index 1 represents the spectroscopic type. This simple formula immediately yields the angle-dependence of the linear dichroism in the oriented trimer system wherein all trimers have parallel planes: $A_{1}\left(\theta_{e}=90^{\circ}\right) / A_{1}\left(\theta_{e}=0^{\circ}\right)=1 / 2 \tan ^{2} \theta_{1}$. The same results were obtained earlier in work by Schirmer and Vincent (27).

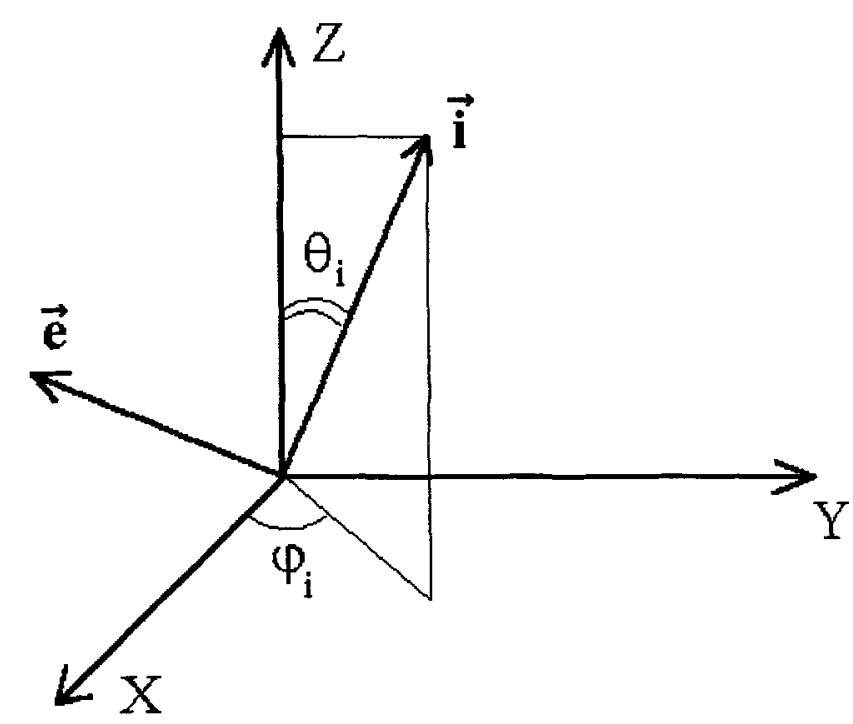

Figure 3. Relative orientations in a Cartesian frame of the polarization of excitation light $\mathbf{e}$ and the transition dipole moment of the chromophore i. The Cartesian frame is rigidly oriented relative to the individual molecular complex, its $\mathrm{Z}$-axis directed along the corresponding symmetry axis. The values $\theta_{i}, \phi_{i}$, are the angular coordinates of the chromophore $i$. 
Using the structural data for the C-PC of the cyanobacteria Mastigocladus laminosus (27), $\theta_{\alpha 84}=78^{\circ}, \theta_{\beta 84}=119^{\circ}$ and $\theta_{\beta 155}=145^{\circ}$; one can calculate the following values of the $A_{\perp} / A_{\mid}$ratio for the $\alpha$ $84, \beta-84$ and $\beta-155$ chromophores, respectively: $11.07,1.63$ and 0.245 . As will be seen below, the latter set of values highlights the fact that the $\beta-155$ chromophores generally have a spatial orientation markedly different from the $\alpha-84$ and $\beta-84$ chromophores, which in general leads to different signs for the linear dichroism (and/or fluorescence polarization) in the case of oriented trimers (28). In the trimer complex all these chromophores have highly overlapping absorption spectra, leading to the spectral dependence of the absorption anisotropy observed by Schirmer and Vincent (27).

The case of fluorescence anisotropy is more complicated, especially if the trimers are randomly oriented in space. Let us first consider the simplified version of a trimer having only three chromophores of the same spectral type (see Fig. 2), i.e. a triple-chromophore complex. Recently Demidov (20) has derived an analytical formula for the degree of fluorescence polarization for such complexes:

$$
\begin{aligned}
\mathbf{P} & =\frac{\mathbf{I}_{11}-\mathbf{I}_{\perp}}{\mathbf{I}_{\| 1}+\mathbf{I}_{\perp}} \\
& =\frac{3\left(\mathbf{q}_{12} \cos ^{2} \theta_{12}+q_{13} \cos ^{2} \theta_{13}+q_{23} \cos ^{2} \theta_{23}\right)-1+2 B}{3+\left(q_{12} \cos ^{2} \theta_{12}+q_{13} \cos ^{2} \theta_{13}+q_{23} \cos ^{2} \theta_{23}\right)+4 B},
\end{aligned}
$$

where

$$
\mathrm{q}_{12}+\mathrm{q}_{13}+\mathrm{q}_{23}=1
$$

Here the indices 1-3 are employed to designate the three chromophores, and $\theta_{\mathrm{ij}}$ means the angle between chromophores $\mathrm{i}$ and $\mathrm{j}$. The parameters $B$ and $q$ represent the rates of energy transfer between chromophores and their spectroscopic parameters (for details see Demidov (20)). When all chromophores are of the same spectral type we obtain the values $q_{12}=q_{13}=q_{23}=1 / 3, B=0.5$ and $\theta_{12}=$ $\theta_{13}=\theta_{23}$. The dependence of these angles of mutual orientation on the angle $\theta$ of inclination of the chromophore transition dipole moment relative to the $\mathrm{Z}$ axis is given by $\cos \theta_{i j}=1 / 2\left(3 \cos ^{2} \theta-1\right)$. Thus we obtain the following formula for monochromophoric trimers:

$$
P=\frac{3 \cos ^{2} \theta_{i j}}{5+\cos ^{2} \theta_{i j}}=\frac{9 \cos ^{4} \theta-6 \cos ^{2} \theta+1}{3 \cos ^{4} \theta-2 \cos ^{2} \theta+7} .
$$

In the particular case where $\theta=90^{\circ}$, i.e. when all three chromophores lie in one plane, this formula yields $\mathrm{P}=1 / 7$, and when $\theta=$ 0 , the value $\mathbf{P}=0.5$. For angles $\theta$ equal to $\theta_{\alpha 84}=78^{\circ}, \theta_{\beta 84}=119^{\circ}$ or $\theta_{\beta 155}=145^{\circ}$ one would obtain degrees of fluorescence polarization equal to $0.11,0.013$ or 0.15 , respectively.

In the case of randomly oriented trimers containing three distinct spectral types of chromophore, i.e. where the total number of chromophores is equal to nine, we have to consider energy redistribution between all the chromophores under specific wavelengths of fluorescence excitation and detection. Generally, determination of the degree of fluorescence polarization involves solution of an energy equilibration system with nine equations and consequent orientational averaging of the solution to account for random orientation of the trimers. Nevertheless, if steady-state excitation is used and the energy migration is faster than the intrinsic rate of chromophore fluorescence $\left(\tau^{-1}\right)$, the energy distribution will be homogeneous over chromophores of the same spectral type. It means, for example, that for chromophores of the first spectral type, all the excitation $\mathrm{A}_{1}$ (Eq. 1) is equally distributed among them. Thus, the original system of nine equations can be reduced to a system of three (29):

$$
\begin{aligned}
& \mathrm{n}_{1}=\mathrm{g}_{11} \mathrm{~A}_{1}+\mathrm{g}_{12} \mathrm{~A}_{2}+\mathrm{g}_{13} \mathrm{~A}_{3} \\
& \mathrm{n}_{2}=\mathrm{g}_{21} \mathrm{~A}_{1}+\mathrm{g}_{22} \mathrm{~A}_{2}+\mathrm{g}_{23} \mathrm{~A}_{3} \\
& \mathrm{n}_{3}=\mathrm{g}_{31} \mathrm{~A}_{1}+\mathrm{g}_{32} \mathrm{~A}_{2}+\mathrm{g}_{33} \mathrm{~A}_{3}
\end{aligned}
$$

where $g_{i j}$ is an element of a matrix whose inverse originates from the system of balance equations (see Appendix 2), the latter equations describing energy redistribution amongst chromophores of the three spectral types. Also in Eq. 4 the parameters $n_{j}$ are the excited state populations of the $\mathrm{j}$-type chromophores ( $\mathrm{j}$ denoting the spectral type).

We have adopted a model entailing a homogeneous distribution of the excitation among chromophores of the same spectral form, such that the probability of finding any one chromophore of spectral type $j$ in its excited state is equal to $1 / 3 n_{j}$. The total intensity of fluorescence emitted from the kth chromophore of spectral type $\mathrm{j}$ is $F_{k j}=1 / 3 p_{k} n_{j}=1 / 3 \eta_{j} \tau_{j}^{-1} n_{j}$, while the component parallel to the polarization of the incident light is $F_{k j}=F_{k j}(k, e)^{2}$. Here $p_{k}$ is the rate of radiative transition and $\eta_{j}$ the fluorescence quantum yield. Following the above procedure we can find the following expression for the net intensity of fluorescence emitted from all chromophores of spectral form $\mathrm{j}$ :

$$
\begin{aligned}
\mathrm{F}^{\mid l} & =\frac{\eta_{j}}{3 \tau_{j}} \sum_{k=1}^{3} n_{j}(\mathbf{k}, \mathbf{e})^{2} \\
& =\frac{\eta_{j}}{\tau_{j}} n_{i}\left(\cos ^{2} \theta_{j} \cos ^{2} \theta_{e}+0.5 \sin ^{2} \theta_{j} \sin ^{2} \theta_{e}\right) \\
F_{j} & =\frac{\eta_{j}}{\tau_{j}} n_{j} .
\end{aligned}
$$

One can immediately see that either the absorption parameters A (Eq. 1) or the fluorescence signals (5) depend solely on the angles $\theta$ and not on the angles $\phi$. This is a result of the symmetrical structure of the C-PC trimer.

We have investigated the reliability of our assumption about the homogeneous distribution of energy among chromophores of identical type. Under steady-state excitation the absorption anisotropył as well as the degree of fluorescence polarization is in each case equal to the result that is obtained for the long-time $(\mathrm{t} \rightarrow \infty)$ polarization kinetics, provided that $\tau^{-1} \ll k_{\mathrm{ij}}$, where $k_{\mathrm{ij}}$ are the rates of energy transfer (see below). The latter condition is certainly fulfilled in the specific complex of C-PC, for example. Thus, in particular, we calculate that at $\mathrm{t}=2 \mathrm{~ns}$ the absorption anisotropy is independent of the angle $\phi$ of the $\beta-155$ chromophores within the C-PC trimers: the uncertainty in such calculations of anisotropy is less than $3 \%$, for a range of $\phi$ values between $0^{\circ}$ and $180^{\circ}$. The precise extent of this uncertainty follows mainly from the accuracy of the numerical calculations involved. In contrast we have found a significant dependence of the absorption anisotropy on the angles $\theta$. Analogous behavior was found for the $\beta-84$ chromophores in the "fictional" trimer consisting of chromophores of two spectral types (see below). In all of these numerical calculations we considered randomly oriented trimers.

The total fluorescence emitted by the analyzed trimer at some particular wavelength $\lambda_{f}$ is given by $F\left(\lambda_{f}\right)=F_{1} f_{1}\left(\lambda_{f}\right)+F_{2} f_{2}\left(\lambda_{f}\right)+$ $F_{3} f_{3}\left(\lambda_{f}\right)$, where $f_{j}\left(\lambda_{f}\right)$ is the fluorescence spectrum of a type $j$ chromophore, normalized in the sense that $\int \mathrm{f}_{\mathrm{i}}(\lambda) \mathrm{d} \lambda=1$. Equations 1,4 and 5 provide the basis for calculation of the fluorescence polarization. The next steps are dependent on the conditions for the conduction of experimental measurements. In the current work we consider two possibilities: (1) trimers with parallel orientation and (2) trimers randomly oriented. The formulas for fluorescence polarization derived below can easily be modified for estimation of the absorption anisotropy $(21,22)$. The resulting formulas will be valid both for trimer and higher aggregates; the only difference is in the absolute values of the $\mathrm{g}_{\mathrm{ij}}$ parameters involved (see Table 1 and following discussion).

\section{TRIMERS WITH PARALLEL ORIENTATION}

First we consider trimers with parallel orientation, by which we mean trimers having a parallel (Z-axis) orientation of their disks. In our calculations we use the spectral parameters of the cyanobacteria $M$. laminosus (see Appendix 1), energy transfer rates given in Table 1 and angles $\theta_{\mathrm{j}}$ reported in the paper by Schirmer and Vincent (27). Three special cases deserve attention.

\$W consider only absorption recovery of the ground state. The excited state absorption and stimulated emission are not considered. 
Table 1. The averaged rates of energy transfer among C-PC chromophores $\left(\mathrm{ns}^{-1}\right)$

\begin{tabular}{lccc}
\hline & \multicolumn{3}{c}{ Acceptor } \\
\cline { 2 - 4 } Donor & $\alpha-84$ & $\beta-84$ & $\beta-155$ \\
\hline Trimer & & & \\
$\alpha-84$ & 2.3 & 1568 & 1.5 \\
$\beta-84$ & 873 & 16 & 8.6 \\
$\beta-155$ & 5 & 44.5 & 1 \\
Hexamer & & & \\
$\alpha-84$ & 361 & 1590 & 26 \\
$\beta-84$ & 895 & 37 & 12 \\
$\beta-155$ & 86 & 62 & 330 \\
Three-four hexamer rod* & & \\
$\alpha-84$ & 375 & 1624 & 26.6 \\
$\beta-84$ & 903 & 145 & 13 \\
$\beta-155$ & 89 & 68 & 336 \\
\hline
\end{tabular}

*The averaged values of energy transfer rates are practically identical for the C-PC rod consisting of three and four hexamers.

Polarization of incident light parallel to the symmetry axis of the trimer

Consider a system of trimers having parallel orientation, excited by light polarized along the axis of symmetry $Z$ (see Fig. 4) such that $\theta_{e}=0$. In the following we shall designate, by the terms parallel and perpendicular, fluorescence components parallel $\left(F_{\|}\right)$and perpendicular $\left(F_{\perp}\right)$ to the polarization of incident light. The application of Eqs. 1, 4 and 5 in this case yields the dependence:

$$
\begin{aligned}
& \xi=\frac{\mathbf{F}_{1}}{\mathbf{F}_{\|}}=\left(\frac{1}{2}\right) \frac{\mu_{1} \sin ^{2} \theta_{1}+\mu_{2} \sin ^{2} \theta_{2}+\mu_{3} \sin ^{2} \theta_{3}}{\mu_{1} \cos ^{2} \theta_{1}+\mu_{2} \cos ^{2} \theta_{2}+\mu_{3} \cos ^{2} \theta_{3}}, \\
& \mathbf{P}=\frac{1-\xi}{1+\xi}
\end{aligned}
$$

where

$$
\begin{aligned}
& \mu_{1}=\eta_{1} \tau_{1}^{-1} f_{1}\left(\lambda_{f}\right) {\left[g_{11} \sigma_{1}\left(\lambda_{a}\right) \cos ^{2} \theta_{1}+g_{12} \sigma_{2}\left(\lambda_{a}\right) \cos ^{2} \theta_{2}\right.} \\
&\left.+g_{13} \sigma_{3}\left(\lambda_{a}\right) \cos ^{2} \theta_{3}\right] \\
& \mu_{2}=\eta_{2} \tau_{2}^{-1} f_{2}\left(\lambda_{f}\right)\left[g_{21} \sigma_{1}\left(\lambda_{a}\right) \cos ^{2} \theta_{1}+g_{22} \sigma_{2}\left(\lambda_{a}\right) \cos ^{2} \theta_{2}\right. \\
&\left.+g_{23} \sigma_{3}\left(\lambda_{a}\right) \cos ^{2} \theta_{3}\right] \\
& \mu_{3}=\eta_{3} \tau_{3}^{-1} f_{3}\left(\lambda_{f}\right)\left[g_{31} \sigma_{1}\left(\lambda_{a}\right) \cos ^{2} \theta_{1}+g_{32} \sigma_{2}\left(\lambda_{a}\right) \cos ^{2} \theta_{2}\right. \\
&\left.+g_{33} \sigma_{3}\left(\lambda_{a}\right) \cos ^{2} \theta_{3}\right] .
\end{aligned}
$$

The particular case of $\mathrm{C}-\mathrm{PC}$ with the spatial orientation under current consideration was experimentally investigated by Priestle et al. (30). They measured a degree of fluorescence polarization $P=-0.17$ at $\lambda_{\mathrm{a}}=568.2 \mathrm{~nm}$. Unfortunately their work is not entirely free from the effects of singletsinglet annihilation and reabsorption and does not contain data about the wavelength of fluorescence detection $\lambda_{\mathrm{f}}$. The latter uncertainty makes it difficult to choose an appropriate value for $\lambda_{\mathrm{f}}$ to compare their experimental data with our theoretical result. Nevertheless, for $\lambda_{f}=700 \mathrm{~nm}$ we calculate a degree of polarization $P=-0.25$, in broad agreement with the experimental data under the given experimental conditions.

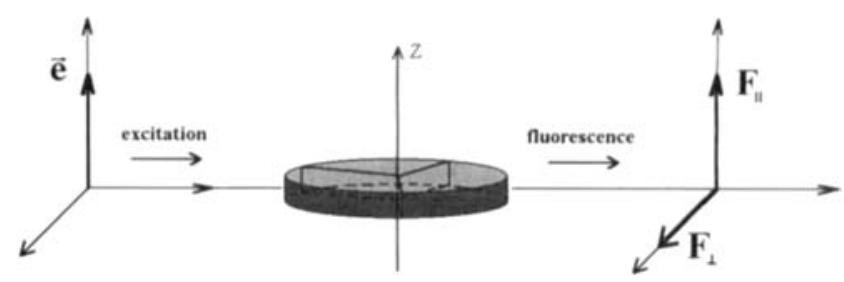

Figure 4. Scheme of relative orientation of a trimer illuminated by polarized light with a polarization e oriented along the $\mathrm{Z}$-axis. The parameters $F_{\|}$and $F_{\perp}$ represent components of fluorescence parallel and perpendicular to the direction of e. All other trimers (not depicted) have their ( $Z$ ) axes of symmetry parallel to that shown.

\section{Polarization of incident light perpendicular to the} symmetry axis of the trimer

We consider the case when the fluorescence is detected along the $\mathrm{Z}$ axis. This yields the result:

$$
\begin{gathered}
F_{\perp}=F_{\|}=\frac{C}{2}\left(\mu_{1} \sin ^{2} \theta_{1}+\mu_{2} \sin ^{2} \theta_{2}+\mu_{3} \sin ^{2} \theta_{3}\right), \\
\xi=\frac{F_{\perp}}{F_{\|}}=1, \quad P=\frac{1-\xi}{1+\xi}=0 .
\end{gathered}
$$

Thus the registered fluorescence is fully depolarized. The result can be compared with the $P$ value of -0.04 experimentally obtained by Priestle et al. (30) for C-PC.

\section{Excitation by unpolarized light}

In the case of excitation by unpolarized light the resulting fluorescence still emerges with a degree of polarization described by Eq. 6, where $F_{\|}$now represents the component parallel to the $\mathrm{Z}$-axis and $F_{\perp}$ the component perpendicular to this axis. The $\mu$ parameters are given by:

$$
\begin{aligned}
& \mu_{1}=\eta_{1} \tau_{1}^{-1} f_{1}\left(\lambda_{\mathrm{f}}\right)\left[g_{11} \sigma_{1}\left(\lambda_{\mathrm{a}}\right)+\mathrm{g}_{12} \sigma_{2}\left(\lambda_{\mathrm{a}}\right)+\mathrm{g}_{13} \sigma_{3}\left(\lambda_{\mathrm{a}}\right)\right] \\
& \mu_{2}=\eta_{2} \tau_{2}^{-1} f_{2}\left(\lambda_{\mathrm{f}}\right)\left[\mathrm{g}_{21} \sigma_{1}\left(\lambda_{\mathrm{a}}\right)+\mathrm{g}_{22} \sigma_{2}\left(\lambda_{\mathrm{a}}\right)+\mathrm{g}_{23} \sigma_{3}\left(\lambda_{\mathrm{a}}\right)\right] \\
& \mu_{3}=\eta_{3} \tau_{3}^{-1} \mathrm{f}_{3}\left(\lambda_{\mathrm{f}}\right)\left[\mathrm{g}_{31} \sigma_{1}\left(\lambda_{\mathrm{a}}\right)+\mathrm{g}_{32} \sigma_{2}\left(\lambda_{\mathrm{a}}\right)+\mathrm{g}_{33} \sigma_{3}\left(\lambda_{\mathrm{a}}\right)\right] .
\end{aligned}
$$

The case of oriented C-PC crystals ( $M$. laminosus) excited by unpolarized light with a wavelength $\lambda_{a}=570 \mathrm{~nm}$ was experimentally investigated by Schirmer and Vincent (27). The results of their measurements and our theoretical calculations based on Eqs. 6 and 9 are presented in Fig. 5. The calculated and measured data are in reasonable agreement, especially if account is taken of the following. Schirmer and Vincent (27) found that "the position of the fluorescence maximum varied between 643 and $655 \mathrm{~nm}$ for different samples" for microcrystals and "between 645 and $662 \mathrm{~nm}$ " for large single crystals "dependent on the crystal size and measuring geometry." They believe that the most significant factor in producing such behavior was a significant amount of reabsorption. When trimers are excited along the $\mathrm{Z}$-axis the theory predicts a value for the polarization of zero, i.e. the C-PC fluorescence must be unpolarized, as experimentally confirmed by Schirmer and Vincent (27).

There is one more factor that complicates comparison of the experimental data on C-PC fluorescence with calculations based on the derived formulas. These formulas are derived subject to the condition that the energy transfer occurs solely inside the molecular complex, such as a trimer, hex- 


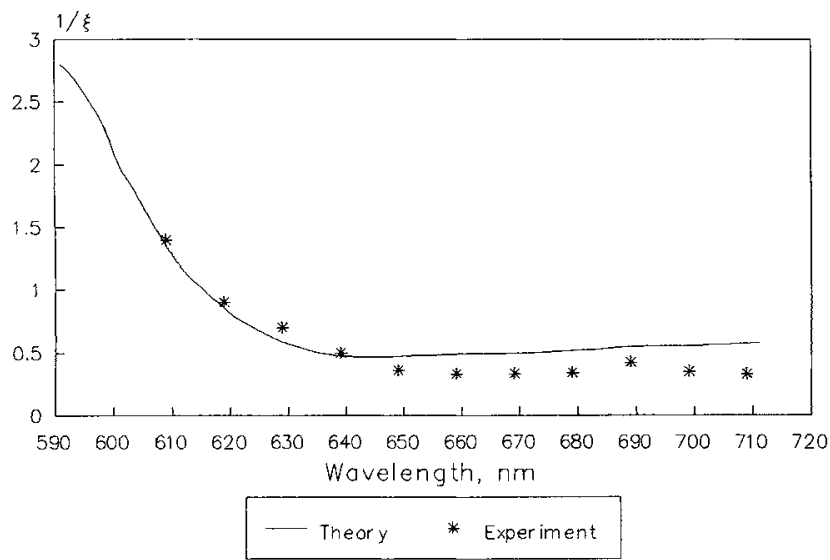

Figure 5. Graph of the spectral dependence of the ratio of fluorescence components $(\mathrm{F})$ parallel and perpendicular to the symmetry axis $Z: \xi^{-1}=F_{1} / F_{1}$. The oriented single crystal of C-PC, consisting of trimers (27), is excited by unpolarized light at a wavelength $\lambda_{a}$ $=570 \mathrm{~nm}$ and fluorescence is detected at a wavelength $\lambda_{\mathrm{f}}=670$ $\mathrm{nm}$. The solid line represents the result of our calculations; the asterisks are the measured values (27)

amer or rod-in other words there is no energy transfer between individual molecular complexes. Obviously this condition can be violated in crystals because of interaggregate energy transfer. In particular, in C-PC crystals one can expect to find more or less effective energy transfer between $\beta-155$ chromophores in adjoining aggregates. The latter chromophores are positioned on the periphery of the trimers, while the $\alpha-84$ and $\beta-84$ chromophores are buried inside (see Fig. 1). On the other hand such intercomplex energy exchange should mainly influence the kinetics of polarization anisotropy, whereas the value measured under steady-state conditions should not be significantly affected. The considered energy migration does not change the symmetry of excitation equilibration between chromophores of similar spectral types but principally the values of $g_{i j}$.

\section{RANDOMLY ORIENTED TRIMERS}

When the trimers are in solution, for example, they are randomly oriented relative to the polarization of incident light. Thus, we have to conduct a rotational average of the fluorescence components, i.e. the average values of the total fluorescence $\langle\mathrm{F}\rangle$ and the component $\left\langle\mathrm{F}_{\|}\right\rangle$parallel to the polarization of the incident light. The averaging yields the following result for $\left.\mathrm{X}=\left\langle\mathrm{F}_{\|}\right\rangle /<\mathrm{F}\right\rangle$ :

$$
\begin{aligned}
\chi=\frac{1}{5}\{ & g_{11}\left(3 \cos ^{4} \theta_{1}+2-2 \cos ^{2} \theta_{1}\right) \\
+ & \gamma_{21} \alpha_{21} g_{22}\left(3 \cos ^{4} \theta_{2}+2-2 \cos ^{2} \theta_{2}\right) \\
+ & \gamma_{31} \alpha_{31} g_{33}\left(3 \cos ^{4} \theta_{3}+2-2 \cos ^{2} \theta_{3}\right) \\
+ & \left(\alpha_{21} g_{12}+\gamma_{21} g_{21}\right) \\
& \cdot\left(3 \cos ^{2} \theta_{2} \cos ^{2} \theta_{1}+2-\cos ^{2} \theta_{2}-\cos ^{2} \theta_{1}\right) \\
+ & \left(\alpha_{31} g_{13}+\gamma_{31} g_{31}\right) \\
& \cdot\left(3 \cos ^{2} \theta_{3} \cos ^{2} \theta_{1}+2-\cos ^{2} \theta_{3}-\cos ^{2} \theta_{1}\right)
\end{aligned}
$$

$$
\begin{aligned}
+ & \left(\alpha_{31} \gamma_{21} g_{23}+\gamma_{31} \alpha_{21} g_{32}\right) \\
& \left.\cdot\left(3 \cos ^{2} \theta_{3} \cos ^{2} \theta_{2}+2-\cos ^{2} \theta_{3}-\cos ^{2} \theta_{2}\right)\right\} \\
\div & \left\{g_{11}+\gamma_{21} g_{21}+\gamma_{31} g_{31}+\alpha_{21}\left(g_{12}+\gamma_{21} g_{22}+\gamma_{31} g_{32}\right)\right. \\
& \left.+\alpha_{31}\left(g_{13}+\gamma_{21} g_{23}+\gamma_{31} g_{33}\right)\right\} .
\end{aligned}
$$

This equation is straightforward, but cumbersome: the degree of fluorescence polarization may be calculated from it in the regular way, through $\mathrm{P}=(3 x-1) /(x+1)$. In Eq. 10 the parameters $\alpha_{i j}$ and $\gamma_{i j}$ are given by $\alpha_{i j}=\sigma_{i}\left(\lambda_{a}\right) / \sigma_{j}\left(\lambda_{a}{ }^{8}\right.$ and $\gamma_{i j}=\left[\eta_{i} f_{i}\left(\lambda_{f}\right) \tau_{j}\right] /\left[\eta_{j} f_{j}\left(\lambda_{f}\right) \tau_{i}\right]$; the angles $\theta_{i}(i=1,2,3)$ are the inclinations of the chromophore transition dipole moments relative to the trimer symmetry axis, and the indexes $i, j$ denote the chromophore spectral types. Equation 10 is valid not only for trimers but also for hexamers and higher order aggregates. Again the difference is only in the values of the $g_{i j}$ parameters.

Equation 10 yields simple values for the special situations where (a) $\theta_{1}=\theta_{2}=\theta_{3}=0$ and (b) $\theta_{1}=\theta_{2}=\theta_{3}=90^{\circ}$. Irrespective of the values of the other parameters involved, case (a) yields $\chi=0.6$ and $P=0.5$, i.e. the result expected for a system of parallel dipoles. Case $b$, which relates to a structure where all dipoles of the individual complex are oriented in the same plane, yields the values $\chi=0.4$ and $P$ $=1 / 7$. The latter result is the same as in the case of the monochrome trimer described above. From Eq. 10 one finds, in the special case where only chromophores of the first spectral type are able to absorb light and fluorescence (i.e. $\alpha_{i 1}=0$ and $\gamma_{i 1}=0$ ), a degree of fluorescence polarization in accordance with Eq. 3. Thus, our current calculations are in agreement with previous work by Demidov (20).

An intermediate case, where the trimer contains only chromophores of two spectral types, can also be analyzed from the formulas derived above. Allophycocyanin $(23,31,32)$ and C-PC of the mutant strain PR6235 (cpcB/C 155S) (33) are particular examples of such complexes. For such cases we have $\alpha_{31}=0, \gamma_{31}=0$ and $\mathrm{g}_{\mathrm{i} 3}=\mathrm{g}_{3 \mathrm{i}}=0$. The latter yields an expression for $\chi$, which is as follows:

$$
\begin{aligned}
x=\frac{1}{5} & \left\{g_{11}\left(3 \cos ^{4} \theta_{1}+2-2 \cos ^{2} \theta_{1}\right)\right. \\
& +\gamma_{21} \alpha_{21} g_{22}\left(3 \cos ^{4} \theta_{2}+2-2 \cos ^{2} \theta_{2}\right) \\
& +\left(\alpha_{21} g_{12}+\gamma_{21} g_{21}\right) \\
& \left.\cdot\left(3 \cos ^{2} \theta_{2} \cos ^{2} \theta_{1}+2-\cos ^{2} \theta_{2}-\cos ^{2} \theta_{1}\right)\right\} \\
\div & \left\{g_{11}+\gamma_{21} g_{21}+\alpha_{21}\left(g_{12}+\gamma_{21} g_{22}\right)\right\}
\end{aligned}
$$

Here $\mathrm{g}_{11}=\mathrm{q}_{22} / \mathrm{D}, \mathrm{g}_{12}=-\mathrm{q}_{12} / \mathrm{D}, \mathrm{g}_{21}=-\mathrm{q}_{21} / \mathrm{D}, \mathrm{g}_{22}=\mathrm{q}_{22} / \mathrm{D}$ and $D=q_{11} q_{22}-q_{12} q_{21}$; for the definition of the $q_{i j}$ parameters see Appendix 2. Equation 11 can be further simplified in certain extreme cases of unidirectional donor-acceptor interaction:

(a) where light is absorbed solely by "donor" molecules $\left(\alpha_{21}=0\right)$ and fluorescence occurs only from "acceptors"' $\left(\gamma_{21}=\infty\right)$, then we have

${ }^{8}$ In experiments the more commonly used parameters are molar extinction coefficients $\epsilon$, measured in $\mathrm{mol} \mathrm{m} \mathrm{m}^{-2}$ units. In terms of these parameters $\alpha_{i j}=\epsilon_{i}\left(\lambda_{a}\right) / \epsilon_{j}\left(\lambda_{a}\right)$. 


$$
x=\frac{1}{5}\left(3 \cos ^{2} \theta_{2} \cos ^{2} \theta_{1}+2-\cos ^{2} \theta_{2}-\cos ^{2} \theta_{1}\right)
$$

giving

$$
\mathbf{P}=\frac{9 \cos ^{2} \theta_{2} \cos ^{2} \theta_{1}-3 \cos ^{2} \theta_{2}-3 \cos ^{2} \theta_{1}+1}{3 \cos ^{2} \theta_{2} \cos ^{2} \theta_{1}-\cos ^{2} \theta_{2}-\cos ^{2} \theta_{1}+7}
$$

for any values of $\mathrm{g}_{\mathrm{ij}}$, i.e., arbitrary $k_{12}, k_{21}, \tau_{\mathrm{i}}$;

(b) for absorption and fluorescence by a single species of donor $\left(\alpha_{21}=0, \gamma_{21}=0\right)$, then

$$
x=\frac{1}{5}\left\{3 \cos ^{4} \theta_{1}+2-2 \cos ^{2} \theta_{1}\right\}
$$

and for absorption and fluorescence entirely by acceptors $\left(\alpha_{21}=\infty, \gamma_{21}=\infty\right)$

$$
x=\frac{1}{5}\left\{3 \cos ^{4} \theta_{2}+2-2 \cos ^{2} \theta_{2}\right\},
$$

yielding formulas for the fluorescence exactly as presented in Eq. 3;

(c) both donors and acceptors can both absorb light and fluoresce, but $k_{12} \gg k_{21} \gg \tau^{-1}$. Here we obtain

$$
\begin{aligned}
\chi= & \frac{1}{5}\left\{\alpha_{21}\left(3 \cos ^{4} \theta_{2}+2-2 \cos ^{2} \theta_{2}\right)\right. \\
& \left.+\left(3 \cos ^{2} \theta_{2} \cos ^{2} \theta_{1}+2-\cos ^{2} \theta_{2}-\cos ^{2} \theta_{1}\right)\right\} \\
& \div\left\{\alpha_{21}+1\right\} .
\end{aligned}
$$

In this last case one can observe that the result, Eq. 16, does not contain the parameter $\gamma_{21}$, and thus the excitation spectrum of polarized fluorescence does not depend on the wavelength of fluorescence detection. The physical explanation for this is that the substantially more efficient flow of energy from chromophores of the first type to the second produces an excitation predominantly located on chromophores of the second type. The latter thus provide the major contribution to the emitted fluorescence, whereas chromophores of the first type do not contribute significantly. An analogous dependence was found in the case of simple double-chromophore complexes (19).

By analysis of a number of experimental studies $(12,23,32,34)$ one can conclude that the APC trimer case is governed by $\mathrm{Eq} .16$, i.e. it is probable that in the APC trimer the absorption and fluorescence spectra of the $\beta$-chromophores are significantly more red-shifted, relative to the $\alpha$ chromophores, than in C-PC. The former APC provides the condition $k_{12} \gg k_{21}$ and obviously $k_{21} \gg \tau^{-1}$. The application of Eq. 16 to the experimental results by Yeh et al. (32) obtained with steady-state excitation provides $\theta_{\alpha} \approx 27^{\circ}$ and $\theta_{\beta} \approx 40^{\circ}$. Yeh et al. (32) found for the short-wavelength band of fluorescence excitation (where $\alpha_{21} \approx 0$ ) a degree of fluorescence polarization $\mathrm{P}$ equal to 0.15 , whereas for the long-wavelength band (where $\alpha_{21} \rightarrow \infty$ ) $\mathrm{P} \cong 0.084$. The question can arise: how accurate are these evaluations of $\theta_{\alpha}$ and $\theta_{\beta}$, depending as they do on the accuracy of the measured degrees of polarization? Unfortunately Yeh et al. (32) did not assess the accuracy of their measurements; nevertheless if this accuracy was, let us say, about $10 \%$, then one can ascertain that the accuracy in calculation of the angles $\theta_{\alpha}$ and $\theta_{\beta}$ is $22 \%$ and $3 \%$, respectively.

By adopting these values for the angles one can reason-

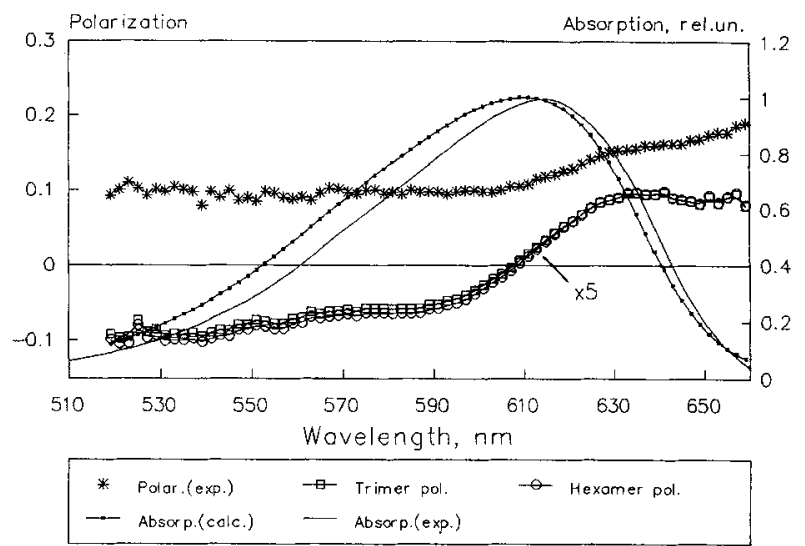

Figure 6. Absorption and excitation spectra of fluorescence polarization for C-PC, calculated and measured by Mimuro et al. (35); wavelength of fluorescence detection $\lambda_{f}=670 \mathrm{~nm}$. Curves marked as $\square \longrightarrow \square$ and $\mathrm{O}-\mathrm{O}$ are calculated.

ably explain the experimental results of Sharkov et al. $(23,34)$ (absorption anisotropy) and Xie et al. (12) (fluorescence anisotropy) obtained with femtosecond excitation. These angles significantly differ from the values (27) $\theta_{\alpha}=$ $78^{\circ}, \theta_{\beta}=119^{\circ}$ (or $\theta_{\beta}=61^{\circ}$, with the same $\cos ^{2}$ value) expected by a number of researchers from the homology between APC and C-PC, and by the additional assumption of an equality between the chromophore inclinations (relative to the $\mathrm{Z}$-axis) in the C-PC crystal and the trimer in solution. However application of the latter angular values yields polarization data that differ significantly from the experimental results obtained in the above-cited works $(12,23,32)$. The fact is that, as yet, nothing definite is known about the chromophore inclination angles in the APC trimer. Thus, we come to the conclusion that this question requires more careful investigation.

\section{Application to C-PC aggregates}

In the following we will conduct a theoretical analysis of the polarization properties of C-PC aggregates by applying the formulas derived above. In our calculations we use the spectral parameters of the cyanobacteria M. laminusos (see Appendix 1), energy transfer rates given in Table 1 and angles $\theta_{\mathrm{j}}$ reported in the paper by Schirmer and Vincent (27). The calculated fluorescence polarization spectra (both for excitation and emission) are presented in Figs. 6 and 7. In addition, these figures present the calculated absorption and fluorescence spectra of the C-PC trimers, as well as experimental data measured by Mimuro et al. (35). In contrast with our previous work on the C-PC $\beta$-subunit and monomer (1921,36 ), we now find a significant difference in the calculated and measured degrees of fluorescence polarization. A difference between the calculated and measured absorption and fluorescence spectra can also be observed, but it is not so dramatic.

We are able to outline a few factors responsible for such differences. Before doing so, however, it is important that the following be noted: the polarization spectra are much more sensitive both to the infrastructure of the molecular complexes and to intracomplex energy migration than the fluorescence and absorption spectra. There have been many 


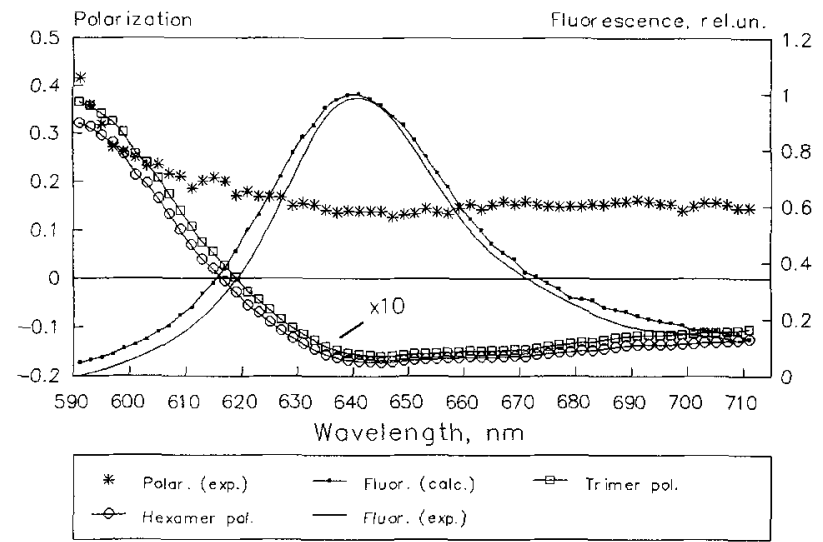

Figure 7. Fluorescence and emission spectra of fluorescence polarization for C-PC, calculated and measured by Mimuro et al. (35); wavelength of excitation $\lambda_{\mathrm{a}}=550 \mathrm{~nm}$. Curves marked as $\square$ $\square$ and $\mathrm{O} \bigcirc$ are calculated.

attempts to calculate the absorption and fluorescence spectra of the C-PC trimer and higher aggregates on the basis of the individual spectra of the $\alpha$ - and $\beta$-chromophores $(27,28)$. Each time the calculated and measured results have differed. The commonly accepted explanation for these differences is that the local protein environment in the higher aggregates is different from the lower aggregates. This yields a difference in the individual spectra of C-PC chromophores upon higher order aggregation from those in the lower aggregates. Unfortunately, there have as yet been no experimental attempts to extract the individual spectra of the C-PC chromophores bound in the higher aggregates. Our work should facilitate such experimental studies by providing the necessary theoretical basis of such a deconvolution.

As noted above, the differences that arise in the case of fluorescence polarization are more dramatic, compared to the absorption and fluorescence spectra, because the polarization spectra are highly sensitive both to the chromophore spectra and the spatial structure of molecular complexes. Again, the fact that there is a lack of experimental work focused on the problem of whether the structure of the C-PC trimers, hexamers etc. are identical in crystals and in solutions, nor yet in vivo or in situ samples. In particular, the peripheral $\beta-155$ chromophores are not so strongly bound with the protein as the inner $\alpha-84$ and $\beta-84$ chromophores-the flexibility of the $\beta-155$ chromophores can therefore result in a change of their orientations in different C-PC structures. Such a flexibility has indeed been mentioned in work by Schirmer et al. (27). Nevertheless even this flexibility is not enough to explain the observed difference between measured and calculated data for the degree of fluorescence polarization. In Fig. 8 we present a three dimensional graph showing the dependence of the degree of fluorescence polarization calculated for different values of $\theta_{\beta 155}$ and $\lambda_{\mathrm{a}}$. Here the values of $\theta_{\beta 84}$ and $\theta_{\mathrm{\alpha} 84}$ are equal to $78^{\circ}$ and $119^{\circ}$, respectively, and the wavelength of fluorescence detection $\lambda_{\mathrm{f}}$ is $670 \mathrm{~nm}$. It transpires that at no angle $\theta_{\mathrm{B} 155}$ can one fit the experimental data $(\mathrm{P}=0.1-$ $0.2)$ with that determined theoretically. Consequently, the choice of values $(27,37)$ for $\theta_{\beta 84}$ and $\theta_{\alpha 84}$ should perhaps be investigated. The latter conclusion about possible flexibility of the internal chromophores coincides with our analysis of two-chromophoric complexes, such as APC, made in the previous section.

The work by Schirmer et al. (27) is one of a few (see also Priestle et al. (30)) devoted to comparison of the spectroscopic properties of C-PC complexes in crystal form and in solution. Unfortunately, these few works are far from complete and do not give an answer to the question stated above. Thus we have to base our calculations on the spatial structure of the C-PC determined for C-PC crystals. We then have to accept the fact that the results of our calculations of fluorescence polarization (as well as absorption anisotropy, see below) highlight the possibility of a difference in the spatial organization of the C-PC trimer, hexamer etc. in crystals and solutions. Such a problem invites further investigation of the C-PC complexes.

\section{EXCITATION BY A $\delta$-PULSE}

In the case of $\delta$-pulse excitation the dynamics of excitation equilibration amongst all the chromophores is very important and includes equilibrium amongst chromophores of identical spectral type. Polarization spectroscopy, in contrast to conventional fluorometry, allows one to trace such interchromophore energy migration among spectroscopically identical chromophores. Thus, in general, we cannot conduct an averaging over chromophores of the same spectral type as for the case of steady-state excitation, and all chromophores must be taken into account at the stage of equilibration.

Let us consider a molecular complex consisting of $\mathrm{N}$ chromophores of three spectral types. Energy migration amongst these chromophores can be described by the following master-equation system:

$$
\begin{gathered}
\frac{\mathrm{d} \mathbf{n}_{1}}{\mathrm{dt}}=-\frac{\mathrm{n}_{1}}{\tau_{\mathrm{l}}}-\sum_{\mathrm{i}=2}^{\mathrm{N}} k_{1 \mathrm{i}} \mathrm{n}_{1}+\sum_{\mathrm{i}=2}^{\mathrm{N}} k_{\mathrm{il}} \mathrm{n}_{\mathrm{i}} \\
\frac{\mathrm{dn} \mathbf{n}_{\mathrm{i}}}{\mathrm{dt}}=-\frac{\mathrm{n}_{\mathrm{j}}}{\tau_{\mathrm{j}}}-\sum_{\substack{\mathrm{i}=1 \\
\mathrm{i} \neq \mathrm{j}}}^{\mathrm{N}} k_{\mathrm{ji}} \mathrm{n}_{\mathrm{j}}+\sum_{\substack{\mathrm{i}=1 \\
\mathrm{i} \neq \mathrm{j}}}^{\mathrm{N}} k_{\mathrm{ij}} \mathrm{n}_{\mathrm{i}} \\
\vdots \\
\frac{\mathrm{dn} \mathbf{n}_{\mathrm{N}}}{\mathrm{dt}}=-\frac{\mathrm{n}_{\mathrm{N}}}{\tau_{\mathrm{N}}}-\sum_{\mathrm{i}=1}^{\mathrm{N}-1} k_{\mathrm{Ni}} \mathbf{n}_{\mathrm{N}}+\sum_{\mathrm{i}=1}^{\mathrm{N}-1} k_{\mathrm{iN}} \mathbf{n}_{\mathrm{i}} .
\end{gathered}
$$

In this system $n_{j}$ is the probability to find chromophore $j$ in the excited state; $\tau_{j}$ is the intrinsic lifetime of its deexcitation, i.e. via any processes other than energy transfer; $k_{\mathrm{ji}}$ is the rate of energy transfer from a chromophore $\mathrm{j}$ to chromophore i. The above master-equation system must be solved under the following initial conditions:

$$
\begin{gathered}
n_{j}(t=0)=\operatorname{Co}\left(\lambda_{a}, j\right)(\mathbf{j}, \mathbf{e})^{2} \\
=\operatorname{C\sigma }\left(\lambda_{a}, j\right)\left[\sin \theta_{j} \sin \theta_{e} \cos \left(\varphi_{j}-\varphi_{e}\right)\right. \\
\left.\quad+\cos \theta_{j} \cos \theta_{e}\right]^{2}, \\
j=1, \ldots, N
\end{gathered}
$$

where

$$
\sigma\left(\lambda_{\mathrm{a}}, \mathrm{j}\right)=\sigma_{1}\left(\lambda_{\mathrm{a}}\right),
$$

if chromophore $\mathrm{j}$ is of the first type, and $\sigma_{2}\left(\lambda_{\mathrm{a}}\right), \sigma_{3}\left(\lambda_{\mathrm{a}}\right)$ appear 


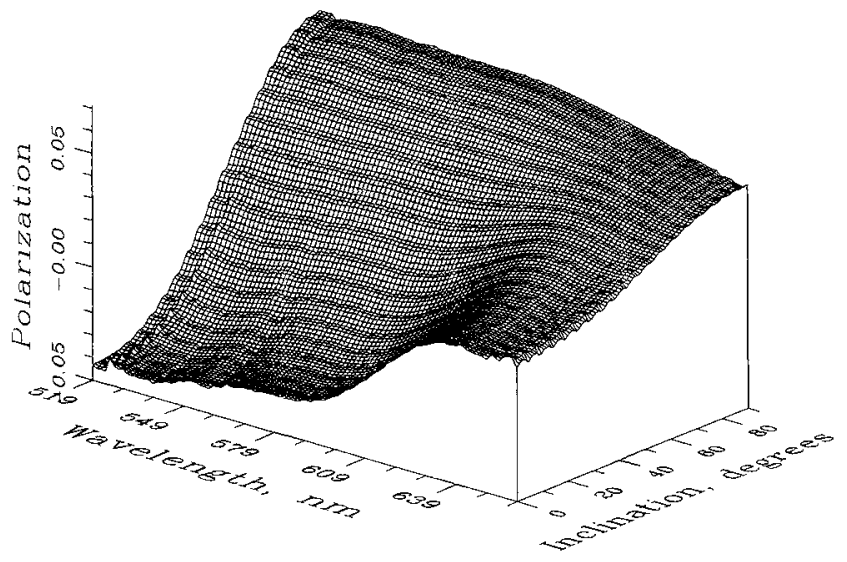

Figure 8. Dependence of the degree of fluorescence polarization of C.PC, $\mathrm{P}(\mathrm{t} \rightarrow \infty) \approx \mathrm{P}(2 \mathrm{~ns})$, on the wavelength $\lambda_{\mathrm{a}}$ and the angle $\theta_{\beta 155}$ of inclination of the $\beta-155$ chromophores relative to symmetry axis $Z$. Inclination angles of the $\alpha-84$ and $\beta-84$ chromophores are $78^{\circ}$ and $119^{\circ}$, respectively. The degree of fluorescence polarization is determined by a computer program designed for calculation of the depolarization kinetics of fluorescence and absorption. Wavelength of fluorescence detection $\lambda_{\mathrm{f}}=670 \mathrm{~nm}$.

on the right for chromophores of the second and third types, respectively. In the case of the $\mathrm{C}-\mathrm{PC}$ trimer the number of equations $N=9$; for the hexamer $N=18$, and for the threehexamer rod, 54. The system (Eq. 17) can be solved by numerical methods involving computers, for example, using the Runge-Cutta method." The resulting function $n_{j}(t), j=$ $\mathrm{I}, \ldots \mathrm{N}$, describes the kinetics of deexcitation of chromophore $\mathrm{j}$. The intensity of fluorescence emitted from such chromophore at wavelength $\lambda_{\mathrm{f}}$ is given by $\mathrm{F}_{\mathrm{j}}\left(\lambda_{\mathrm{f}}, \mathrm{t}\right)=$ $\eta_{j} \tau_{j}^{-1} f\left(\lambda_{f}, j\right) n_{j}(t)$, where the fluorescence spectrum function $\mathbf{f}\left(\lambda_{\mathrm{f}}, \mathbf{j}\right)$ is

$$
\left.\begin{array}{rl}
f\left(\lambda_{\mathrm{f}}, \mathrm{j}\right) & =\mathrm{f}_{1}\left(\lambda_{\mathrm{f}}\right) \\
\eta_{\mathrm{j}} & =\eta_{\mathrm{l}} \\
\tau_{\mathrm{j}} & =\tau_{1}
\end{array}\right\}
$$

if chromophore $\mathrm{j}$ is of the first type, again with corresponding expressions for chromophores of the second and third spectral types, as above. The polarization of the fluorescence emitted by chromophore $\mathrm{j}$ is parallel to its transition dipole moment $\mathbf{j}$; thus the component parallel to the polarization of incident light $e$ is given by $F_{j}\left(\lambda_{f}, t\right)=F_{j}\left(\lambda_{f}, t\right)(\mathbf{j}, e)^{2}$. We consider a solution with a large number of such complexes, and there is no energy transfer between them. Obviously, the overall fluorescence intensity $F\left(\lambda_{f}\right)$ and its component $\mathbf{F}_{\|}\left(\lambda_{f}\right)$ are the sums of $F_{j}\left(\lambda_{f}, t\right)$ and $F_{j} l\left(\lambda_{f}, t\right)$. The procedure of summation depends on the orientations of the chromophore complexes relative to the polarization of incident light. In the current work we will analyze the case of random orientation only.

\section{Aggregates containing identical chromophores}

The degenerate case of monochromophoric trimers, as discussed above, is presented in Fig. 2-this is the special in-

We have developed our own program for such calculations. The program is written on Fortran-77 and is about 20 kilobytes large. It also calculates $\mathbf{k}_{\mathrm{ij}}$ for any given molecular structure.

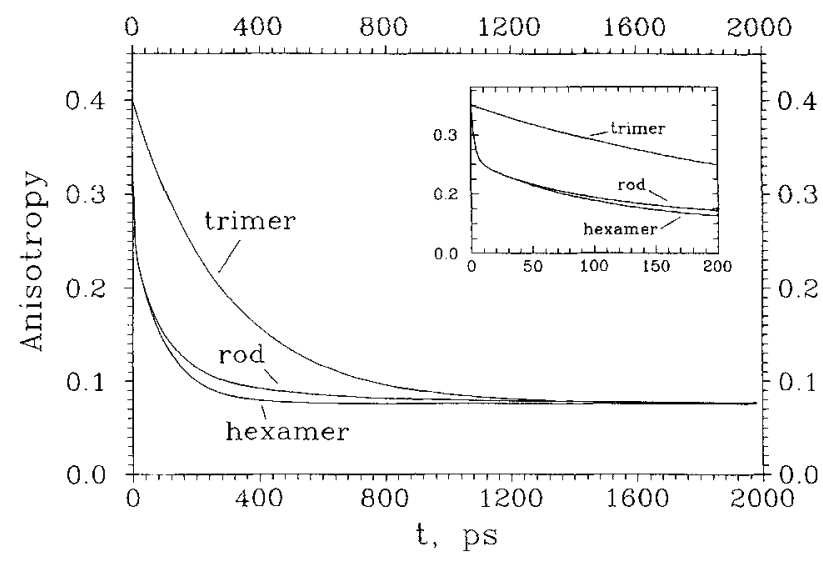

Figure 9. Calculated kinetics of absorption anisotropy of monochromophoric complexes-trimer, hexamer and three-hexamer rod-probed by a $\delta$-pulse having wavelength $\lambda_{a}=615 \mathrm{~nm}$. The inclination angle and spectroscopic parameters of the chromophores are equal to those of the $\alpha-84$ chromophores of C-PC (see text).

stance of a triple-chromophore complex (21) in which the chromophores are identical. In such a case the number of equations in 17 decreases to three and all the $\alpha$ and $\gamma$ parameters are equal to 1 ; moreover the symmetry dictates that all interchromophore energy transfer rates are equal. The resulting formula for the polarization anisotropy, for either fluorescence or absorption, is as follows:

$$
\begin{aligned}
r(t)= & {\left[\frac{1}{10}\left(1-6 \cos ^{2} \theta+9 \cos ^{4} \theta\right)\right] } \\
& +\left[\frac{1}{10}\left(3+6 \cos ^{2} \theta-9 \cos ^{4} \theta\right)\right] \exp (-3 k t)
\end{aligned}
$$

In this equation $k$ is the rate of energy transfer from one chromophore to any other. An analogous dependence $r(t)=$ $a+b e^{-3 k t}$ was previously obtained by Lyle and Struve (18): Eq. 21 discloses that both the $a$ and $b$ parameters depend only on the inclination angle $\theta$, which is consistent with the result of Causgrove et al. (38). One can find that at $\mathrm{t}=0$ the anisotropy $\mathrm{r}=0.4$ for any $\theta$, i.e. the chromophores can be considered quasi-independent. At $t \rightarrow \infty$ the anisotropy is

$$
r(\infty)=1 / 10\left(9 \cos ^{4} \theta-6 \cos ^{2} \theta+1\right) .
$$

It can be observed that $r(\infty)$ is equal to the anisotropy $r$ in the case of steady-state excitation, see Eq. 3, and take into account the dependence $r=2 P /(3-P)$ : an analogous result was obtained in our previous works (19-22).

Unfortunately, the anisotropy for the monochromophoric hexamer and rod do not produce a result as simple as Eq. 21 , and numerical calculations and averaging must be performed on the solution of the master equations. Nevertheless, the analysis shows that the values of anisotropy in the extremes $\mathrm{t}=0$ and $\mathrm{t} \rightarrow \infty$ are identical for different aggregates (trimer, hexamer and rod), i.e. $\mathrm{r}(0)=0.4$ and $\mathrm{r}(\infty)=$ r(steady-state) - see Eq. 22. The latter extreme again depends only on the inclination angle $\theta$. The numerically calculated absorption anisotropy for the above aggregates is presented in Figs. 9-11." We used "pump" and "probe"

IThe analytical Eq. 21 provides kinetics identical to the numerical calculations. The former data are not presented in Figs. 9-11. 


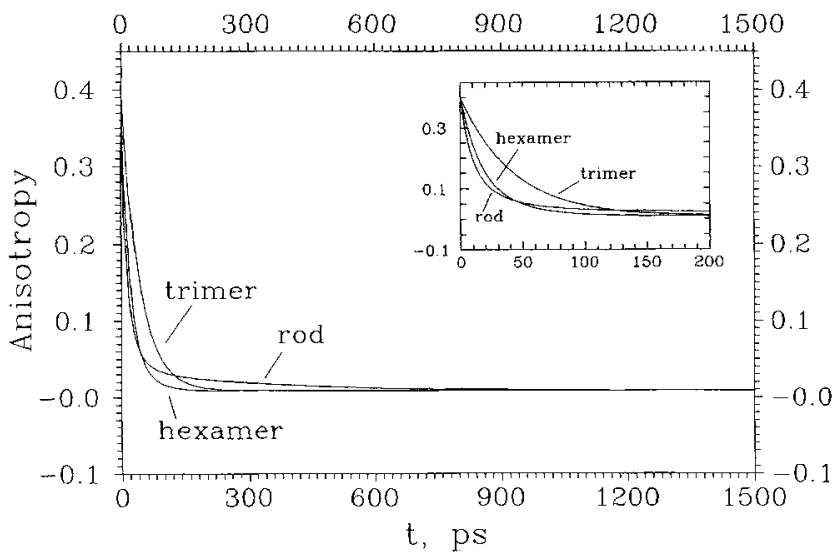

Figure 10. Calculated kinetics of absorption anisotropy of monochromophoric complexes-trimer, hexamer and three-hexamer rod-probed by a $\delta$-pulse having wavelength $\lambda_{\mathrm{a}}=615 \mathrm{~nm}$. The inclination angle and spectroscopic parameters of the chromophores are equal to those of the $\beta-84$ chromophores of C-PC (see text).

pulses with the same wavelength: $\lambda_{\mathrm{a}}=\lambda_{\mathrm{pr}}=615 \mathrm{~nm}$. In our calculations we used the following values for the angles $\theta$ : $\theta_{\alpha 84}=78^{\circ}, \theta_{\beta 84}=119^{\circ}$ and $\theta_{\beta 155}=145^{\circ}$. As predicted by the analytical solution (Eq. 21), the kinetics of the polarization anisotropy for the trimer are monoexponential, whereas for higher aggregates we observe more complex behavior.

In the case of a trimer containing chromophores of type $\mathrm{i}$, the energy transfer rate $k_{\mathrm{i}}$ can be determined in Table 1 from the correspondent diagonal parameter: $k_{\mathrm{i}}=1 / 2 k_{\mathrm{ij}}(\mathrm{i}=1$, 2,3 ). The presence of the factor of a half can be explained as follows. The energy transfer rates presented in Table 1 are calculated as averaged rates of energy withdrawal from a molecule of some particular spectral type. For example, in the case of energy transfer between chromophores in a $\beta-84$ trimer each chromophore has two acceptors, the other $\beta-84$ chromophores, and the pairwise energy transfer rate is thus twice smaller.

In the case of higher aggregates the diagonal elements in the energy transfer matrices cannot be related by any simple analytical formula and are best employed for qualitative evaluation. Thus, for example, it is clear that one should expect a faster decay of the anisotropy with higher values for the energy transfer rates (compare the kinetics in Figs. 9-11 with the corresponding energy transfer rates in Table 1). It was shown by Demidov and Borisov (39) that increasing (C-PC) aggregation causes the appearance of some specific and efficient channels for energy migration.

\section{Aggregates containing chromophores of two spectral types}

In this part we will consider aggregates containing chromophores of two spectral types. The steady-state excitation of such complexes was analyzed above (see Eq. 11). The APC aggregates are examples of such complexes $(12,23)$; a trimer of APC contains six chromophores, and thus the master system involves six equations. Here, it is technically possible to derive an analytical formula, but the result even for the trimer case is too unwieldy to be of practical value. Thus, it is reasonable to employ numerical calculations. For such evaluations we adopt fictional complexes of C-PC aggre-

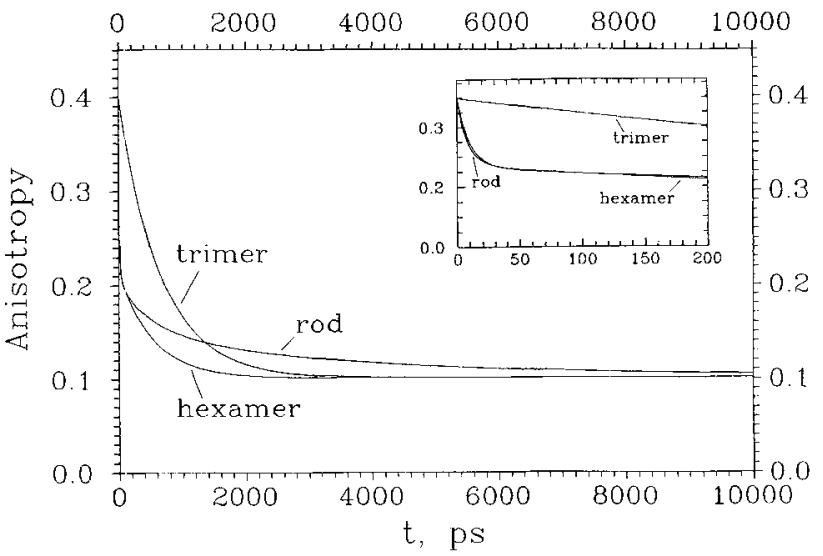

Figure 11. Calculated kinetics of absorption anisotropy of monochromophoric complexes-trimer, hexamer and three-hexamer rod-probed by a $\delta$-pulse having wavelength $\lambda_{\mathrm{a}}=615 \mathrm{~nm}$. The inclination angle and spectroscopic parameters of the chromophores are equal to those of the $\beta-155$ chromophores of C-PC (see text).

gates containing only $\alpha-84$ and $\beta-84$ chromophores. The mutant strain PR6235 (cpcB/C1555) (33) can be considered as a natural example of such a complex. The result of our calculations is presented in Fig. 12. One can observe that the principal features of the polarization anisotropy are analogous to those described in the previous section: (a) an increase of the anisotropy decay rate with increasing C-PC aggregation; (b) $r(0)=0.4$ and $r(\infty) \cong r$ (steady-state); (c) a value for $\mathrm{r}(\infty)$, which is the same for different aggregates.

In view of its potential utility for structure elucidation, it is interesting to consider the sensitivity of polarization features to chromophore orientation. The complexity of the results is such that only a few of the more striking features will be identified in this study-our main aim is to expose the principal dynamic features of the system. In particular, the numerical calculations confirm that at the initial stages of energy equilibration both angles $\theta$ and $\phi$ strongly affect the kinetics of depolarization (Fig. 13a-c). Over longer times, when equilibration is accomplished, the value of the polarization anisotropy depends only on the angle $\theta$, al-

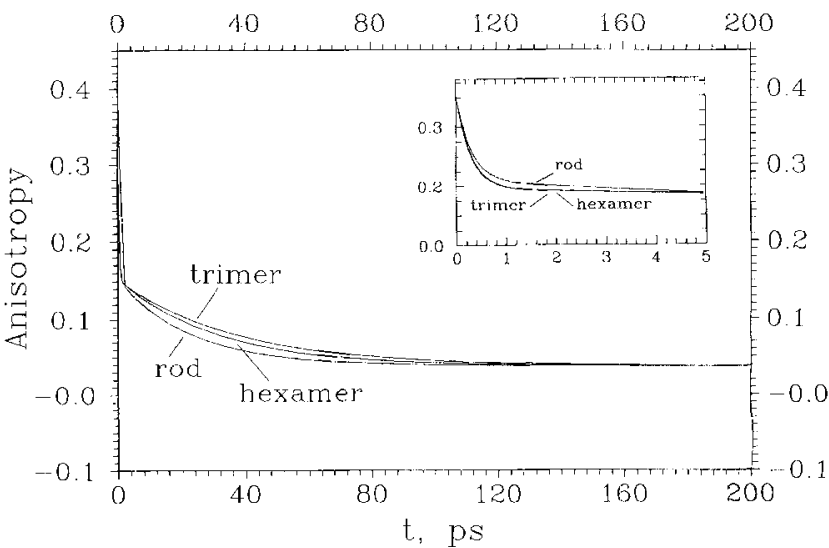

Figure 12. Calculated kinetics of absorption anisotropy of bichromophoric complexes-trimer, hexamer and three-hexamer rodprobed by a $\delta$-pulse having wavelength $\lambda_{a}=615 \mathrm{~nm}$. The inclination angles and spectroscopic parameters of the chromophores are equal to those of the $\alpha-84$ and $\beta-84$ chromophores of C-PC (see text). 

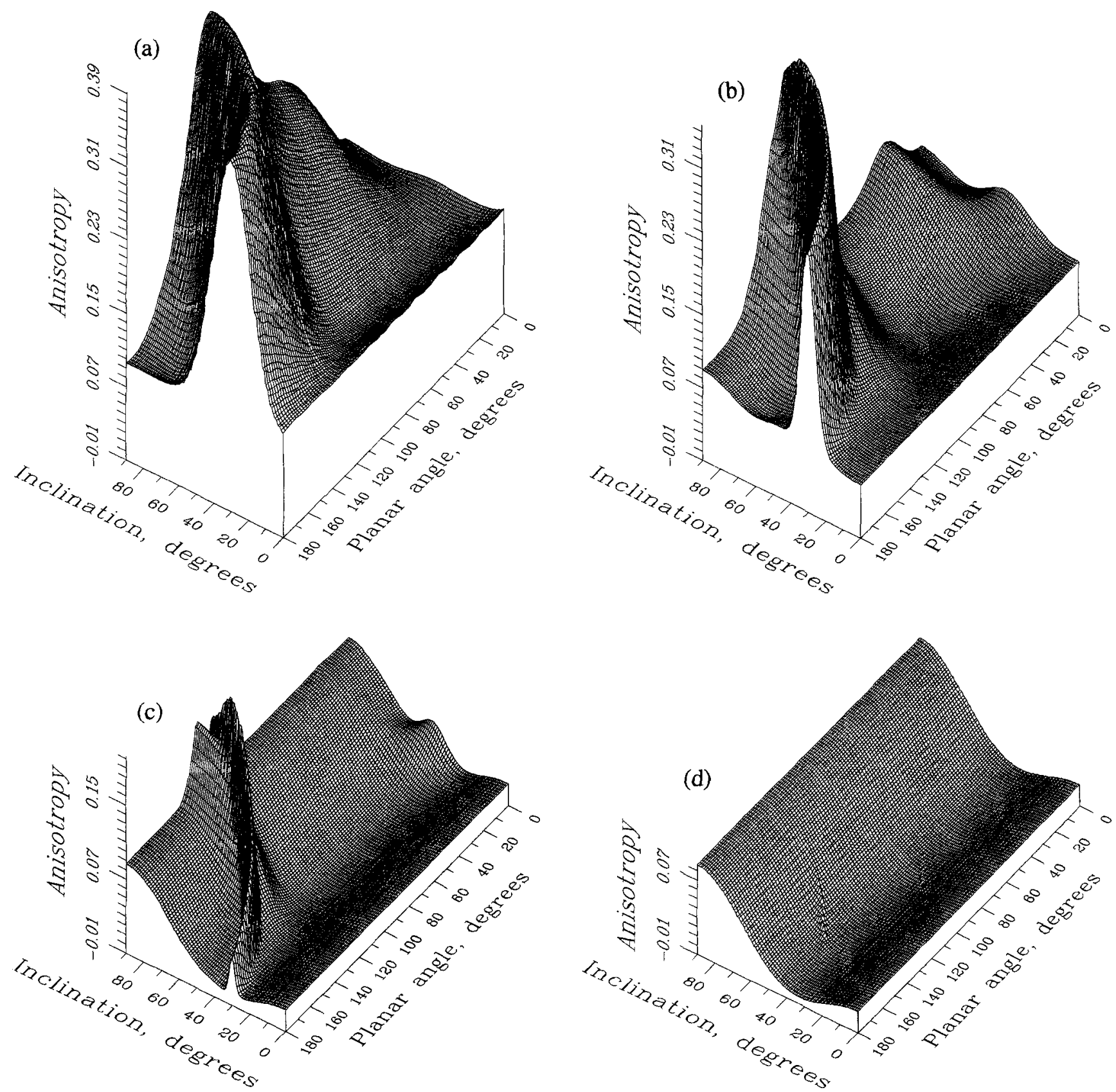

Figure 13. Dependence of the value of absorption anisotropy of bichromophoric trimers on the angular parameters (inclination $\theta$ and planar angle $\phi$ ) of the $\beta-84$ chromophore in the first monomer following excitation by a $\delta$-pulse with wavelength $\lambda_{\mathrm{a}}=615 \mathrm{~nm}$ : (a) $5 \mathrm{ps,} \mathrm{(b)} 30 \mathrm{ps}$, (c) $200 \mathrm{ps}$, (d) $2000 \mathrm{ps}$ after excitation. The spectroscopic parameters of the chromophores are equal to those for the $\alpha-84$ and $\beta-84$ chromophores of C-PC (see text). The planar angles of other $\alpha-84$ chromophores in the trimer are $\phi \pm 120^{\circ}$ and all have the same inclination angle $\theta$, whereas the angles of the $\alpha-84$ chromophores are those determined by Schirmer et al. (37) for C-PC.

though less significantly than at earlier stages. With time, the $\phi$-dependence vanishes; only a vestigial dependence remains after 2 ns, as shown in Fig. 13d, and later this completely disappears. The subnanosecond evolution displays a number of other aspects, as for example in the faster decay of polarization for certain chromophore angles as opposed to others.

At this point the following question can be posed: according to the Förster theory, the energy transfer rates and thus the matrix elements analogous to the $\mathrm{g}_{\mathrm{ij}}$ involved in Eq.
11 are dependent on an orientation factor"; the latter is a function of both angles $\theta$ and $\phi$, so why should the behavior of $r(\infty) \cong r(2 n s)$ be less complicated (see Fig. 13) than could be expected from Eq. 11? The answer is that, under these conditions, (1) a balance of energy distribution has been

"As mentioned earlier, the complete set of energy transfer rates involved in the master-equation system $(17)$ is calculated by our Fortran program for each particular special structure, including angles $\theta$ and $\phi$. 
achieved, which is inversely proportional to the ratio of energy transfer rates; (2) the forward and backward energy transfer rates are identically dependent on the orientation factor, and thus their ratio is free from this parameter. This situation is also reflected in the analytical result under the steady-state excitation-Eq. 11: one can find there that the parameter $\chi$, and hence the polarization anisotropy, is dependent on the ratios $\mathrm{g}_{22} / \mathrm{g}_{11} \cong k_{12} / k_{21}, \mathrm{~g}_{12} / \mathrm{g}_{11} \cong 1$ and $\mathrm{g}_{21} /$ $\mathrm{g}_{11} \cong k_{12} / k_{21}$, when $k_{12}, k_{21} \gg \tau_{1}^{-1}, \tau_{2}^{-1}$.

The fast initial decay of anisotropy with a rate about 400 fs (Fig. 12) is related to the very fast equilibration of energy between neighboring $\alpha-84$ and $\beta-84$ chromophores (see Fig. 1 and Table 1 for the average rates $\left.k_{\alpha 84 \rightarrow \beta 84}, k_{\beta 84 \rightarrow \alpha 84}\right)$. In the case currently under consideration, chromophores of both spectral types can efficiently absorb light and the rates $k_{\alpha 84 \rightarrow \beta 84}, k_{\beta 84 \rightarrow \alpha 84}$ differ relatively little in the different aggregates; moreover the forward and backward transfer rates are also comparable in magnitude.

In the APC-like case (see related discussion earlier) we have $k_{\alpha \rightarrow \beta} \gg k_{\beta \leftarrow \alpha}$, a consequence of the significantly more separated spectra for the $\alpha$ - and $\beta$-chromophores. Sharkov et al. (23) have measured an absorption anisotropy $(\mathrm{r}(0)=$ $0.4)$ very slightly decaying on the $0-4$ ps timescale $\left(\lambda_{\mathrm{a}}=\lambda_{\mathrm{pr}}\right.$ $=620 \mathrm{~nm}$ ). This result can be explained by the fact that at this wavelength it is mainly $\beta$-chromophores that absorb light, i.e. the system has the features of a quasimonochromophoric trimer consisting of $\beta$-chromophores. This type of trimer is considered above and has a behavior similar to that observed experimentally. Xie et al. (12) have made femtosecond measurements of fluorescence polarization in a different spectral range, $\lambda_{\mathrm{a}}=640 \mathrm{~nm}$ and $\lambda_{\mathrm{f}}=730 \mathrm{~nm}$, where the $\beta$-chromophores presumably both absorb light and fluorescence. This again equates to the quasimonochromophoric case and is consistent with the slight decay of fluorescence anisotropy, which was indeed observed in the experiment. Studies by the same authors (12) of the case where $\lambda_{\mathrm{a}}=605$ $\mathrm{nm}$ and $\lambda_{\mathrm{f}}=650 \mathrm{~nm}$ most likely relates to the opposite situation where it is mainly the $\alpha$-chromophores that absorb light. Moreover, as outlined above, we then have $k_{\alpha \rightarrow \beta} \gg$ $k_{\beta \leftarrow \alpha}$. It does not matter in what proportion the $\alpha$ - and $\beta$ chromophores participate in fluorescence. See the discussion, relating to Eqs. 13 and 16 , in the case of steady-state excitation above. In addition, we have performed numerical calculations that have validated this remark. This case yields a very fast decay of fluorescence anisotropy from $r(0)=0.4$, as found in the cited work.

\section{Aggregates containing chromophores of three spectral types}

This is the most complicated case considered in our work. It represents, inter alia, the case of natural C-PC aggregates. Evidently, it is a case for which numerical analysis is the obvious choice. The most complex case of a three-hexamer $\operatorname{rod}$ (with $\mathrm{N}=54$ in Eqs. 17-20) involves about 5 days of continuous calculation on the Unix station Alpha DEC-3000/ 600 to obtain the kinetics of polarization anisotropy over the interval 0-200 ps.

One particular result of our calculations is presented in Fig. 14, which also shows for comparison the experimental data obtained by Gillbro et al. (10). In fact, experimental

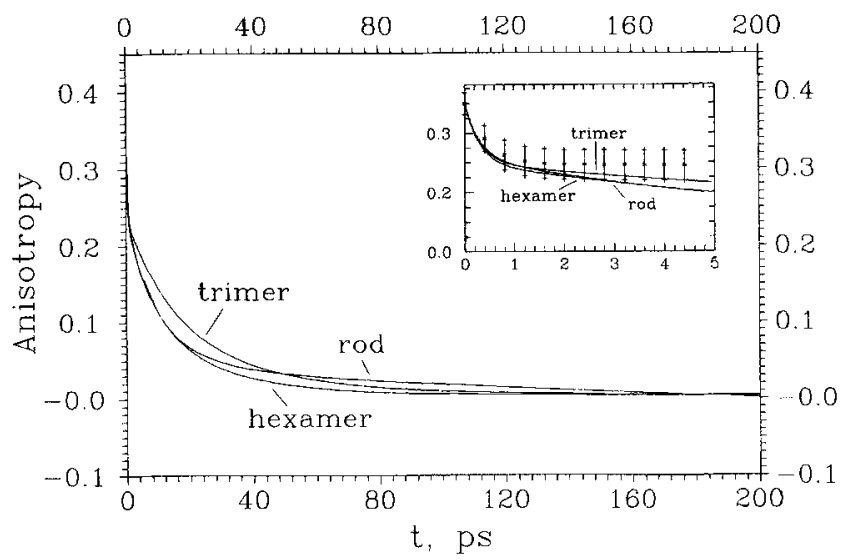

Figure 14. Calculated kinetics of absorption anisotropy for C-PCtrimer, hexamer and three-hexamer rod-probed by a $\delta$-pulse having wavelength $\lambda_{a}=615 \mathrm{~nm}$. The inclination angles and spectroscopic parameters of the chromophores are equal to those determined by Schirmer et al. $(27,37)$ for cyanobacteria $M$. laminosus. On the inset showing the initial behavior, the experimental data of Gillbro et al. (10) are represented by asterisks with the appropriate error bars.

kinetics results are available only for C-PC trimers $(10,12,40,42)$; higher aggregates have not yet been fully investigated. We can identify satisfactory agreement between the experimental data and our calculations over the interval $0-5$ ps (Fig. 14). On the other hand, thr : is a significant difference at much longer times (the experimental data for this range are not presented in Fig. 14 and can be found in the works referred to above). According to experimental measurements the value $r(\infty)$ is about 0.1 , coinciding with the anisotropy measured under steady-state excitation. At the same time our calculation provides a much lower value of $\mathrm{r}(\infty) \cong-0.01$ (anisotropy of fluorescence polarization at $\lambda_{\mathrm{a}}$ $=550 \mathrm{~nm}$ and $\lambda_{\mathrm{f}}=670 \mathrm{~nm}$, not presented in figures), which in turn coincides with our analytical result for steady-state excitation (see Figs. 6, 7). We believe that the observed difference between the measured and numerically calculated values of polarization anisotropy has the same origin as discussed earlier in the case of steady-state excitation. A significant exception to this major disagreement between our calculations and experimental data is the result obtained by Priestle et al. (30), who measured $\mathrm{P}=0.03$ (or $\mathrm{r}=0.02$ ) for the degree of fluorescence polarization of C-PC in solution. This result is the closest to our calculations.

The cause of the fast decay of polarization anisotropy, again with a rate of about $400 \mathrm{fs}$, is the fast energy exchange between $\alpha-84$ and $\beta-84$ chromophores, exactly analogous to that described in the previous section. The difference here lies in the absolute values of polarization anisotropy, higher in the current case because of the contribution of the $\beta-155$ chromophores. The rates of energy flow to and from the $\beta$ 155 chromophores are much slower than others. Thus on the $0-5$ ps timescale the $\beta-155$ chromophores can be considered quasi-independent, retaining their own anisotropy of about 0.4 . The resulting anisotropy of the system can be evaluated as the weighted sum of anisotropies of the $\beta-155$ chromophores and the $\alpha-84-\beta-84$ chromophore complex. Consequently, in the considered time interval the polarization anisotropy provided by the $\beta-155$ chromophores is not particularly sensitive to the angle $\theta_{\beta i 55}$. 


\section{CONCLUSION}

Our theoretical investigation targets the polarization properties of molecular complexes with threefold $\left(C_{3}\right)$ rotational symmetry. Our aim was a derivation of analytical formulas for estimation of the degree of fluorescence polarization and absorption anisotropy for such complexes under steady-state and $\delta$-pulse excitation. Numerical calculations were employed in some particular of cases $\delta$-pulse excitation where the analytical analysis is too cumbersome. The derived formulas have general importance and can be applied for a large variety of $\mathrm{C}_{3}$ molecular complexes, for example, various trimers, hexamers etc. Such complexes can be widely found in biological systems.

In our work we have used C-PC aggregates for practical testing and application of the formulas derived, though not with the aim of conducting a detailed investigation of their structure. Although such a more comprehensive analysis can be essayed in the future, it has sufficed for our present purposes to use data obtained elsewhere. Nevertheless, the analysis of C-PC aggregate polarization data conducted here discloses the necessity for a reappraisal of some common assumptions about the C-PC structure.

Molecular complexes with a rigid and highly organized infrastructure exhibit significant differences in their spectroscopic behavior from unstructured complexes. In the former, one can find significant values for the polarization of fluorescence (or absorption recovery), both under steady-state and $\delta$-pulse excitation, owing to the correlation between the transition dipole moments of the constituent chromophores. By contrast, in unstructured ensembles, the presence of energy transfer efficiently destroys polarization. Thus, polarization data may provide invaluable information for analyzing the structure of molecular complexes. We believe that the formulas derived in the current work, as well as the numerical analysis of the basic behavior of the considered molecular aggregates, will prove useful for further investigations of $\mathrm{C}_{3}$ molecular ensembles.

Acknowledgement--We are pleased to acknowledge the use of ad vanced computer facilities offered by Dr. Andrew Hemmings (UEA).

\section{APPENDIX 1}

\section{Spectroscopic data for the C-PC chromophores}

To compute the polarization spectra of the C-PC aggregates we need to know (1) the fluorescence and absorption spectra of the C-PC chromophores, (2) their intrinsic lifetimes, (3) their fluorescence quantum yields, (4) their spatial orientations $\left\{\theta_{i}, \phi_{i}\right\}$ relative to the trimer axes and (5) the rates of energy transfer between them.

In our calculations we used the chromophore spectra of the C-PC monomer recently determined by Demidov and Mimuro (36). The chromophore fluorescence quantum yields were determined in the same work: $\eta_{\alpha 84}=0.72, \eta_{\beta 84}=0.42$ and $\eta_{\beta 155}$ $=0.96$. The fluorescence lifetimes were borrowed from the work by Sauer and Scheer (43): $\tau_{\alpha 84}=\tau_{\beta 84}=\tau_{\beta 155}=\tau=1.5$ ns. The orientations of the chromophores in the C-PC trimers and hexamers are borrowed from Schirmer et al. (27,37). These data were analyzed and extended for the case of C-PC rods consisting of the same kind of hexamers, to provide the spatial orientation of all chromophores in C-PC rods. The average values of energy transfer rates for the C-PC aggregates were earlier estimated by Demidov and Borisov $(29,39)$ and corrected for new C-PC spectroscopic parameters estimated by Demidov and Mimuro (36), see Table 1.

In the case of $\delta$-pulse excitation we have to use a complete set of energy transfer rates, and we thus have to calculate the rates $k_{\mathrm{ij}}(\mathrm{i}, \mathrm{j}=1, \ldots \mathrm{N}$ ) of transfer between pair of chromophores $i$ and $j$ in the analyzed C-PC aggregate. For this purpose we assumed the Förster mechanism for energy transfer (44), with Förster radiuses (39), the spatial positions and orientations of the C-PC chromophores (37) and the matrix of overlap integrals (36) as given elsewhere. The orientation of the absorption and emission transition dipole moments within each individual chromophore are assumed to be parallel.

\section{APPENDIX 2}

Under steady-state excitation, the excitation populations $n_{j}(i$ $=1,2,3$ ) and energy migration within each individual chromophore complex, see Eq. 4, are determined by the system $\mathbf{Q n}+\mathbf{A}=0$, where $\mathbf{n}$ is the vector of chromophore excitation populations, $\boldsymbol{A}=\left\{\mathrm{A}_{1}, \mathrm{~A}_{2}, \mathrm{~A}_{3}\right\}$ is the vector of excitation conditions proportional to $\sigma_{\mathrm{j}}\left(\lambda_{\mathrm{a}}\right)$, see Eq. 1 , and $\mathbf{Q}$ is the following rate matrix:

$$
\begin{aligned}
\mathbf{Q} & =\left\|q_{\mathrm{jij}}\right\| \\
& =\left\|\begin{array}{ccc}
-\tau_{1}^{-1}-k_{12}-k_{13} & k_{21} & k_{31} \\
k_{12} & -\tau_{2}^{-1}-k_{21}-k_{23} & k_{32} \\
k_{13} & k_{23} & -\tau_{3}^{-1}-k_{31}-k_{32}
\end{array}\right\| .
\end{aligned}
$$

The matrix equation can be solved for the vector $\mathbf{n}$, as given by: $\mathbf{n}=-\mathbf{G A}$, i.e., $\mathrm{n}_{\mathbf{i}}=-\Sigma_{\mathrm{j}} \mathbf{g}_{\mathrm{ij}} \mathrm{A}_{\mathrm{j}}$. The matrix $\left\|\mathbf{g}_{\mathrm{j}}\right\|$ is the inverse of $\left\|q_{i j}\right\|, \mathbf{G Q}=\mathbf{E}$, where $\mathbf{E}$ is the unit (identity) ma trix. The $\mathrm{g}_{\mathrm{ij}}$ elements are given by:

$$
\begin{aligned}
\mathrm{g}_{11} & =\left(\mathrm{q}_{22} \mathrm{q}_{33}-\mathrm{q}_{23} \mathrm{q}_{32}\right) / \mathrm{D} \\
& =\left[\left(\tau_{2}^{-1}+k_{23}+k_{21}\right)\left(\tau_{3}^{-1}+k_{31}+k_{32}\right)-k_{32} k_{23}\right] / \mathrm{D} \\
\mathrm{g}_{12} & =\left(\mathrm{q}_{13} \mathrm{q}_{32}-\mathrm{q}_{12} \mathrm{q}_{33}\right) / \mathrm{D} \\
& =\left[k_{31} k_{23}+k_{21}\left(\tau_{3}^{-1}+k_{31}+k_{32}\right)\right] / \mathrm{D} \\
\mathrm{g}_{13} & =\left(\mathrm{q}_{12} \mathrm{q}_{23}-\mathrm{q}_{22} \mathrm{q}_{13}\right) / \mathrm{D} \\
& =\left[k_{21} k_{32}+k_{31}\left(\tau_{2}^{-1}+k_{23}+k_{21}\right)\right] / \mathrm{D} \\
\mathrm{g}_{21} & =\left(\mathrm{q}_{31} \mathrm{q}_{23}-\mathrm{q}_{21} \mathrm{q}_{33}\right) / \mathrm{D} \\
& =\left[k_{13} k_{32}+k_{12}\left(\tau_{3}^{-1}+k_{31}+k_{32}\right)\right] / \mathrm{D} \\
\mathrm{g}_{22} & =\left(\mathrm{q}_{11} \mathrm{q}_{33}-\mathrm{q}_{31} \mathrm{q}_{13}\right) / \mathrm{D} \\
& =\left[\left(\tau_{1}^{-1}+k_{12}+k_{13}\right)\left(\tau_{3}^{-1}+k_{31}+k_{32}\right)-k_{13} k_{31}\right] / \mathrm{D} \\
\mathrm{g}_{23} & =\left(\mathrm{q}_{21} \mathrm{q}_{13}-\mathrm{q}_{11} \mathrm{q}_{23}\right) / \mathrm{D} \\
& =\left[k_{12} k_{31}+k_{32}\left(\tau_{1}^{-1}+k_{12}+k_{13}\right)\right] / \mathrm{D} \\
\mathrm{g}_{31} & =\left(\mathrm{q}_{21} \mathrm{q}_{32}-\mathrm{q}_{22} \mathrm{q}_{31}\right) / \mathrm{D} \\
& =\left[k_{12} k_{23}+k_{13}\left(\tau_{2}^{-1}+k_{21}+k_{23}\right)\right] / \mathrm{D} \\
\mathrm{g}_{32} & =\left(\mathrm{q}_{12} \mathrm{q}_{31}-\mathrm{q}_{11} \mathrm{q}_{32}\right) / \mathrm{D} \\
& =\left[k_{21} k_{13}+k_{23}\left(\tau_{3}^{-1}+k_{12}+k_{13}\right)\right] / \mathrm{D}
\end{aligned}
$$




$$
\begin{aligned}
\mathrm{g}_{33}= & \left(\mathrm{q}_{11} \mathrm{q}_{22}-\mathrm{q}_{21} \mathrm{q}_{12}\right) / \mathrm{D} \\
= & {\left[\left(\tau_{1}^{-1}+k_{12}+k_{13}\right)\left(\tau_{2}^{-1}+k_{21}+k_{23}\right)-k_{12} k_{21}\right] / \mathrm{D} } \\
\mathrm{D}= & \mathrm{q}_{11}\left(\mathrm{q}_{22} \mathrm{q}_{33}-\mathrm{q}_{23} \mathrm{q}_{32}\right)-\mathrm{q}_{12}\left(\mathrm{q}_{21} \mathrm{q}_{33}-\mathrm{q}_{31} \mathrm{q}_{23}\right) \\
& +\mathrm{q}_{13}\left(\mathrm{q}_{21} \mathrm{q}_{32}-\mathrm{q}_{31} \mathrm{q}_{22}\right) .
\end{aligned}
$$

These $\mathrm{g}_{\mathrm{ij}}$ parameters are used in calculations of the degree of fluorescence polarization under steady-state excitation.

\section{REFERENCES}

1. Craver, F. W. and R. S. Knox (1971) Theory of polarization quenching by excitation transfer. II. Anisotropy and secondneighbour considerations. Mol. Phys. 22, 385-402.

2. Dale, R. E. and J. Eisinger (1974) Intramolecular distances determined by energy transfer. Dependence on orientational freedom of donor and acceptor, Biopolymers 13, 1573-1605.

3. PuJlerits, T. and A. Freiberg (1992) Kinetic-model of primary energy-transfer and trapping in photosynthetic membranes. Biophys. J. 63, 879-896.

4. Andrews, D. L. (1989) A unified theory of radiative and radiationless molecular-energy transfer. Chem. Phys. 135, 195-201.

5. Andrews, D. L. and G. Juzeliünas (1991) The range dependence of fluorescence anisotropy in molecular-energy transfer. $J$. Chem. Phys. 95, 5513-5518.

6. Levshin, V. L. (1925) Polarized fluorescence and phosphorescence of dye solutions. Zh. Rus. Fiz.-Chim. Obschestva, Fizika (USSR) 57, 283-300.

7. Perrin, F. (1929) La fluorescence des solutions. Ann. Phys. 12, $169-275$.

8. Perrin, F. (1925) Theorie de la fluorescence polarise (influence de la viscosite). C. $R$. 180, 581-583.

9. Galli, C., K. Wynne, S. M. LeCours, M. J. Therien and R. M. Hochstrasser (1993) Direct measurement of electronic dephasing using anisotropy. Chem. Phys. Lett. 206, 493-499.

10. Gillbro, T., A. V. Sharkov, I. V. Kryukov, E. V. Khoroshilov, P. G. Kryukov, R. Fischer and H. Scheer (1993) Förster energy transfer between neighbouring chromophores in C-phycocyanin trimers. Biochim. Biophys. Acta 1140, 321-326.

11. Du, M., X. L. Xie, Y. W. Jia, L. Mets and G. R. Fleming (1993) Direct observation of ultrafast energy transfer in PSI core antenna. Chem. Phys. Lett. 201, 535-542.

12. Xie, X., M. Du, L. Mets and G. R. Fleming (1992) Femtosecond fluorescence depolarization study of photosynthetic antenna proteins: observation of ultrafast energy transfer in trimeric C-phycocyanin and allophycocyanin. In Time-Resolved Laser Spectroscopy in Biochemistry III, vol. 1640, pp. 690-706. SPIE.

13. Rahman, T. S., R. S. Knox and V. M. Kenkre (1979) Theory of depolarization of fluorescence in molecular pairs. Chem. Phys. 44, 197-211.

14. Wynne, K. and R. M. Hochstrasser (1993) Coherence effects in the anisotropy of optical experiments. Chem. Phys. 171, 179188.

15. Knox, R. S. and D. Güllen (1993) Theory of polarized fluorescence from molecular pairs: Förster transfer at large electronic coupling. Photochem. Photobiol. 57, 40-43.

16. van Amerongen, H. and W. S. Struve (1992) Dynamic and structural implications of polarized fluorescence spectroscopy in oriented uniaxial antennae. Application of C-phycocyanin from Mastigocladus laminosus. J. Luminesc. 51, 29-38.

17. van Amerongen, H. and W. S. Struve (1995) Polarized optical spectroscopy of chromoproteins. Methods Enzymal. 246, 259 283.

18. Lyle, P. A. and W. S. Struve (1991) Dynamic linear dichroism in chromoproteins. Photochem. Photobiol. 53, 359-365.

19. Demidov, A. A. (1994) Determination of fluorescence polarization of double-chromophore complexes. J. Theor. Biol. 170, 355-358.

20. Demidov, A. A. (1994) Fluorescence polarization of triple-chromophore complexes with energy transfer. Appl. Opt. 33, 63036306.

21. Demidov, A. A. (1994) Quantitative calculations of fuorescence polarization and absorption anisotropy kinetics of double- and triple-chromophore complexes with energy transfer. Biophys. $J$. 67, 2184-2190.

22. Demidov, A. A. and D. L. Andrews (1995) Theory of polarized fluorescence and absorption in molecular complexes comprising two chromophores with non-parallel absorption and emission transition dipole moments. Chem. Phys. Lett. 235, 327-333.

23. Sharkov, A. V., I. V. Kryukov, E. V. Khoroshilov, P. G. Kryukov, R. Fischer, H. Scheer and T. Gillbro (1992) Femtosecond energy transfer between chromophores in allophycocyanin trimers. Chem. Phys, Lett. 191, 633-638.

24. Andrews, D. L. and P. Allcock (1995) Polarisation analysis of bimolecular excitations mediated by energy transfer: a common theoretical framework for fluorescence migration and Raman scattering. Chem. Phys. 198, 35-51.

25. Gantt, E. (1981) Phycobilisomes. Annu. Rev. Plant Physiol. 32, 327-347.

26. Glazer, A. N. (1984) Phycobilisome. A macromolecular complex optimized for light energy transfer, Biochim. Biophys. Acta 768, 29-51.

27. Schirmer, T. and M. G. Vincent (1987) Polarized absorption and fluorescence spectra of single crystals of C-phycocyanin. Biochim. Biophys. Acta 893, 379-385.

28. Juszczak, L. J., B. A. Zilinskas, N. E. Geacintov, J. Breton and K. Sauer (1991) Orientation and linear dichroism of Mastigocladus laminosus phycocyanin trimer and Nostoc sp. phycocyanin dodecamer in stretched poly(vinyl alcohol) films. Biochim. Biophys. Acta 1058, 363-373.

29. Demidov, A. A. and A. Yu. Borisov (1994) Computer simulation of exciton jumping statistics and energy flow in C-phycocyanin of algae Agmenellum quadruplicatum in the presence of traps. Photochem. Photobiol, 60, 46-52.

30. Priestle, J. P., Jr., R. H. Rhyne, Jr., J. B. Salmon and M. L. Hackert (1982) Phycobiliproteins: comparison of solution and single crystal fluorescence for C-phycocyanin and B-phycoerythrin. Photochem. Photobiol. 35, 827-834.

31. Maxson, P., K. Sauer and A. N. Glazer (1988) Fluorescence spectroscopy of allophycocyanin complexes from Synechococcus 6301 strain AN I 2. In Photosynthetic Light-Harvesting Systems. Organization and Function (Edited by $\mathrm{H}$. Scheer and $\mathrm{S}$. Schneider), pp. 439-449. Walter de Gruyter, Berlin, New York.

32. Yeh, S. W., A. N. Glazer and J. H. Clark (1986) Control of bilin transition dipole moment direction by macromolecular assembly: energy transfer in allophycocyanin. J. Phys. Chem. 90 , $4578-4580$.

33. Debreczeny, M., K. Sauer, J. Zhou and D. A. Bryant (1993) Monomeric C-phycocyanin at room temperature and $77 \mathrm{~K}$ : resolution of the absorption and fuorescence spectra of the individual chromophores and the energy-transfer rate constants. $J$. Phys. Chem. 97, 9852-9862.

34. Sharkov, A. V., I. V. Kryukov, E. V. Khoroshilov, P. G. Kryukov, R. Fischer, H. Scheer and T. Gillbro (1994) Femtosecond spectral and anisotropy study of excitation-energy transfer between neighboring $\alpha-80$ and $\beta-81$ chromophores of allophycocyanin trimers. Biochim. Biophys. Acta 1188, 349-356.

35. Mimuro, M., P. Füglistaller, R. Rümbeli and H. Zuber (1986) Functional assignment of chromophores and energy transfer in C phycocyanin isolated from the thermophilic cyanobacterium Mastigocladus laminosus. Biochim. Biophys. Acta 848, 155 166.

36. Demidov, A. A. and M. Mimuro (1995) Deconvolution of Cphycocyanin $\beta-84$ and $\beta-155$ chromophore absorption and fluorescence spectra of cyanobacterium Mastigocladus laminosus. Biophys. J. 68, 1500-1506.

37. Schirmer, T., W. Bode and R. Huber (1987) Refined three-dimensional structures of two cyanobacterial C-phycocyanins at 2.1 and $2.5 \AA$ resolution. J. Mol. Biol. 196, 677-695.

38. Causgrove, T. P., S. Yang and W. S. Struve (1988) Polarized pump probe spectroscopy of exciton transport in bacteriochlorophyll a-protein from Prosthecochloris aestuarii. J. Phys. Chem. 92, 6790-6795.

39. Demidov, A. A. and A. Yu. Borisov (1993) Computer simulation of energy migration in the C-phycocyanin of the blue-green algae Agmenellum quadruplicatum. Biophys. J. 64, 1375-1384. 
40. Sandström, Å, T. Gillbro, U. Sundström, R. Fischer and H. Scheer (1988) Picosecond time-resolved energy transfer within C-phycocyanin aggregates of Mastigocladus laminosus. Biochim. Biophys. Acta 933, 42-53.

41. Xia, A. D., J. C. Zhu, L. J. Jiang, D. L. Li and X. Y. Zhang (1991) Energy transfer kinetics in C-phycocyanin from cyanobacterium Westiellopsis prolofica studied by pump-probe techniques. Biochem. Biophys. Res. Commun. 179, 558-564.

42. Xia, A., J. Zhu, H. Wu, L. Jiang, X. Zhang, M. Sudha and P.
S. M. Sai (1993) Time-resolved polarized absorption of C-phycocyanin from the cyanobacterium Westiellopsis. J. Photochem. Photobiol. B Biol. 19, 111-117.

43. Sauer, K. and H. Scheer (1988) Excitation transfer in C-phycocyanin-Förster transfer rate and exciton calculations based on new crystal-structure data for C-phycocyanins from Agmenellum quadruplicatum and Mastigocladus laminosus. Biochim. Biophys. Acta 936, 157-170.

44. Förster, Th. (1948) Zwischenmolekulare Energiewanderung und Fluoreszenz. Ann. Physik. 6, 55-75. 